\title{
Source-Channel Coding Theorems for the Multiple-Access Relay Channel
}

\author{
Yonathan Murin, Ron Dabora, and Deniz Gündüz
}

\begin{abstract}
We study reliable transmission of arbitrarily correlated sources over multiple-access relay channels (MARCs) and multiple-access broadcast relay channels (MABRCs). In MARCs only the destination is interested in reconstructing the sources, while in MABRCs both the relay and the destination want to reconstruct them. In addition to arbitrary correlation among the source signals at the users, both the relay and the destination have side information correlated with the source signals. Our objective is to determine whether a given pair of sources can be losslessly transmitted to the destination for a given number of channel symbols per source sample, defined as the sourcechannel rate. Sufficient conditions for reliable communication based on operational separation, as well as necessary conditions on the achievable source-channel rates are characterized. Since operational separation is generally not optimal for MARCs and MABRCs, sufficient conditions for reliable communication using joint source-channel coding schemes based on a combination of the correlation preserving mapping technique with SlepianWolf source coding are also derived. For correlated sources transmitted over fading Gaussian MARCs and MABRCs, we present conditions under which separation (i.e., separate and stand-alone source and channel codes) is optimal. This is the first time optimality of separation is proved for MARCs and MABRCs.
\end{abstract}

Index Terms-Multiple-access relay channel, separation theorem, Slepian-Wolf source coding, fading, joint source and channel coding, correlation preserving mapping.

\section{INTRODUCTION}

The multiple-access relay channel (MARC) models a network in which several users communicate with a single destination with the help of a relay [1]. The MARC is a fundamental multi-terminal channel model that generalizes both the multiple access channel (MAC) and the relay channel models, and has received a lot of attention in the recent years [1], [2], [3], [4]. If the relay terminal also wants to decode the source messages, the model is called the multiple-access broadcast relay channel (MABRC).

Previous work on MARCs considered independent sources at the terminals. In the present work we allow arbitrary correlation among the sources to be transmitted to the destination in a

Yonathan Murin and Ron Dabora are with the Department of Electrical and Computer Engineering, Ben-Gurion University, Israel; Email: \{moriny, ron\}eee.bgu.ac.il. Deniz Gündüz is with the Department of Electrical and Electronic Engineering, Imperial College London, London, United Kingdom; Email: d.gunduz@imperial.ac.uk. This work was partially supported by the European Commission's Marie Curie IRG Fellowship PIRG05-GA-2009-246657 under the Seventh Framework Programme. Deniz Gündüz was partially supported by the Spanish Government under project TEC2010-17816 (JUNTOS) and the European Commission's Marie Curie IRG Fellowship with reference number 256410 (COOPMEDIA). Parts of this work were presented at the International Symposium on Wireless Communication Systems (ISWCS), Aachen, Germany, November 2011, and at the International Symposium on Information Theory (ISIT), Boston, MA, July 2012 . lossless fashion, and also let the relay and the destination have side information correlated with the sources. Our objective is to determine whether a given pair of sources can be losslessly transmitted to the destination for a specific number of channel uses per source sample, which is defined as the source-channel rate.

In [5] Shannon showed that a source can be reliably transmitted over a point-to-point memoryless channel, if its entropy is less than the capacity of the channel. Conversely, if the source entropy is greater than the channel capacity, reliable transmission of the source over the channel is not possible. Hence, a simple comparison of the rates of the optimal source code and the optimal channel code for the respective source and channel, suffices to determine whether reliable communication is feasible or not. This is called the separation theorem. An implication of the separation theorem is that the independent design of the source and the channel codes is optimal. However, the optimality of source-channel separation does not generalize to multiuser networks [6], [7], [8], and, in general the source and the channel codes need to be designed jointly for every particular combination of sources and channel.

The fact that the MARC generalizes both the MAC and the relay channel models reveals the difficulty of the problem studied here. The capacity of the relay channel, which corresponds to a special case of our problem, is still unknown. While the capacity region of a MAC is known in general, the optimal joint source-channel code for transmission of correlated sources over the MAC remains open [7]. Accordingly, the objective of this work is to construct lower and upper bounds for the achievable source-channel rates in MARCs and MABRCs. We shall focus on decode-and-forward (DF) based achievability schemes, such that the relay terminal decodes both source signals before sending cooperative information to the destination. Naturally, DF-based achievable schemes for the MARC directly apply to the MABRC model as well. Moreover, we characterize the optimal source-channel rate in some special cases. Our contributions are listed below:

1) We establish an achievable source-channel rate for MARCs based on operational separation [10, Section I]. The scheme uses the DF strategy with irregular encoding [9], [2, Section I-A], successive decoding at the relay and backward decoding at the destination. We show that for MARCs with correlated sources and side information, DF with irregular encoding yields a higher achievable source-channel rate than the rate achieved by DF with regular encoding. This is in contrast to the scenario without side information, in which DF with regular encoding achieve the same source-channel rate as DF with irregular encoding. The achievability result 
obtained for MARCs applies directly to MABRCs as well.

2) We derive two sets of necessary conditions for the achievability of source-channel rates for MARCs (and MABRCs).

3) We investigate MARCs and MABRCs subject to independent and identically distributed (i.i.d.) fading, for both phase fading and Rayleigh fading. We find conditions under which informational source-channel separation (in the sense of $[10$. Section I]) is optimal for each channel model. This is the first time the optimality of separation is proven for some special case of MARCs and MABRCs. Note that these models are not degraded in the sense of [11].

4) We derive two joint source-channel coding achievability schemes for MARCs and MABRCs for the source-channel rate $\kappa=1$. Both proposed schemes use a combination of SlepianWolf (SW) source coding [12] and joint source-channel coding implemented via the correlation preserving mapping (CPM) technique [7]. In the first scheme CPM is used for encoding information to the relay and SW source coding combined with an independent channel code is used for encoding information to the destination. In the second scheme, SW source coding is used for encoding information to the relay and CPM is used for encoding information to the destination. These are the first joint source-channel achievability schemes, proposed for a multiuser network with a relay, which take advantage of the CPM technique.

\section{Prior Work}

The MARC has been extensively studied from a channel coding perspective. Achievable rate regions for the MARC were derived in [2], [3] and [13]. In [2] Kramer et al. derived an achievable rate region for the MARC with independent messages. The coding scheme employed in [2] is based on decode-and-forward relaying, and uses regular encoding, successive decoding at the relay, and backward decoding at the destination. In [3] it was shown that, in contrast to the classic relay channel, in a MARC different DF schemes yield different rate regions. In particular, backward decoding can support a larger rate region than sliding window decoding. Another DF-based coding scheme which uses offset encoding, successive decoding at the relay, and sliding-window decoding at the destination was presented in [3]. Outer bounds on the capacity region of MARCs were obtained in [13]. More recently, capacity regions for two classes of MARCs were characterized in [4].

In [14], Shamai and Verdú considered the availability of correlated side information at the receiver in a point-topoint scenario, and showed that source-channel separation still holds. The availability of correlated side information at the receiver enables transmitting the source reliably over a channel with a smaller capacity compared to the capacity needed in the absence of side information. In [7] Cover et al. derived finite-letter sufficient conditions for communicating discrete, arbitrarily correlated sources over a MAC, and showed the suboptimality of source-channel separation when transmitting correlated sources over a MAC. These sufficient conditions were later shown in [15] not to be necessary in general. The transmission technique introduced by Cover et al. is called correlation preserving mapping $(C P M)$. In the CPM technique the channel codewords are correlated with the source sequences, resulting in correlated channel inputs. CPM is extended to source coding with side information over a MAC in [16] and to broadcast channels with correlated sources in [17] (with a correction in [18]).

In [10] Tuncel distinguished between two types of sourcechannel separation. The first type, called informational separation, refers to classical separation in the Shannon sense. The second type, called operational separation, refers to statistically independent source and channel codes, which are not necessarily the optimal codes for the underlying source or the channel, coupled with a joint decoder at the destination. Tuncel also showed that when broadcasting a common source to multiple receivers, each with its own correlated side information, operational separation is optimal while informational separation is not.

In [8] Gündüz et al. obtained necessary and sufficient conditions for the optimality of informational separation in MACs with correlated sources and side information at the receiver. The work [8] also provided necessary and sufficient conditions for the optimality of operational separation for the compound MAC. Transmission of arbitrarily correlated sources over interference channels (ICs) was studied in [19], in which Salehi and Kurtas applied the CPM technique; however, when the sources are independent, the conditions derived in [19] do not specialize to the Han and Kobayashi (HK) region, [20], which is the largest known achievable rate region for ICs. Sufficient conditions based on the CPM technique, which specialize to the HK region were derived in [21]. Transmission of independent sources over ICs with correlated receiver side information was studied in [22]. The work [22] showed that source-channel separation is optimal when each receiver has access to side information correlated with its own desired source. When each receiver has access to side information correlated with the interfering transmitter's source, [22] provided sufficient conditions for reliable transmission based on a joint source-channel coding scheme which combines Han-Kobayashi superposition encoding and partial interference cancellation.

Lossless transmission over a relay channel with correlated side information was studied in [23], [24], [25] and [26]. In [23] Gündüz and Erkip developed a DF-based achievability scheme and showed that operational separation is optimal for physically degraded relay channels as well as for cooperative relay-broadcast channels. The scheme of [23] was extended to multiple relay networks in [24].

Prior work on source transmission over fading channels is mostly limited to point-to-point channels (see [27] and references therein). In this work we consider two types of fading models: phase fading and Rayleigh fading. Phase fading models apply to high-speed microwave communications where the oscillator's phase noise and the sampling clock jitter are the key impairments. Phase fading is also the major impairment in communication systems that employ orthogonal frequency division multiplexing [28]. Additionally, phase fading can be used to model systems which employ dithering to decorrelate signals [29]. For cooperative multi- 
user scenarios, phase-fading models have been considered for MARCs [2], [13], [31], for broadcast-relay channels (BRCs) [2], and for interference channels [32]. Rayleigh fading models are very common in wireless communications and apply to mobile communications in the presence of multiple scatterers without line-of-sight [30]. Rayleigh fading models have been considered for relay channels in [33], [34] and [35], and for MARCs in [31]. The key similarity between the two fading models is the uniformly distributed phase of the fading process. The phase fading and the Rayleigh fading models differ in the behavior of the fading magnitude component, which is fixed for the former but varies following a Rayleigh distribution for the latter.

The rest of this paper is organized as follows: in Section (II) the model and notations are presented. In Section [III an achievable source-channel rate based on operational separation is presented. In Section IV necessary conditions on the achievable source-channel rates are derived. In Section $\nabla$ the optimality of separation for correlated sources transmitted over fading Gaussian MARCs is studied, and in Section VI two achievable schemes based on joint source-channel coding are derived. Concluding remarks are provided in Section VII followed by the appendices.

\section{NOTATIONS AND MODEL}

In the following we denote the set of real numbers with $\mathfrak{R}$, and the set of complex numbers with $\mathfrak{C}$. We denote random variables (RVs) with upper-case letters, e.g. $X, Y$, and their realizations with lower case letters, e.g. $x, y$. A discrete $\mathrm{RV} X$ takes values in a set $\mathcal{X}$. We use $|\mathcal{X}|$ to denote the cardinality of a finite, discrete set $\mathcal{X}, p_{X}(x)$ to denote the probability mass function (p.m.f.) of a discrete $\mathrm{RV} X$ over $\mathcal{X}$, and $f_{X}(x)$ to denote the probability density function (p.d.f.) of a continuous $\mathrm{RV} X$ on $\mathfrak{C}$. For brevity we may omit the subscript $X$ when it is the uppercase version of the sample symbol $x$. We use $p_{X \mid Y}(x \mid y)$ to denote the conditional distribution of $X$ given $Y$. We denote vectors with boldface letters, e.g. $\mathbf{x}, \mathbf{y}$; the $i$ 'th element of a vector $\mathbf{x}$ is denoted by $x_{i}$, and we use $\mathbf{x}_{i}^{j}$ where $i<j$, to denote $\left(x_{i}, x_{i+1}, \ldots, x_{j-1}, x_{j}\right) ; x^{j}$ is a short form notation for $x_{1}^{j}$, and unless specified otherwise, $\mathbf{x} \triangleq$ $x^{n}$. We denote the empty set with $\phi$, and the complement of the set $\mathcal{B}$ by $\mathcal{B}^{c}$. We use $H(\cdot)$ to denote the entropy of a discrete $\mathrm{RV}$, and $I(\cdot ; \cdot)$ to denote the mutual information between two RVs, as defined in [36, Ch. 2, Ch. 9]. We use $A_{\epsilon}^{*(n)}(X)$ to denote the set of $\epsilon$-strongly typical sequences with respect to the distribution $p_{X}(x)$ on $\mathcal{X}$, as defined in [37. Ch. 5.1]; when referring to a typical set we may omit the $\mathrm{RV}$ s from the notation, when these variables are clear from the context. We use $\mathcal{C N}\left(a, \sigma^{2}\right)$ to denote a proper, circularly symmetric, complex Gaussian distribution with mean $a$ and variance $\sigma^{2}$ [38], and $\mathbb{E}\{\cdot\}$ to denote stochastic expectation. We use $X-Y-Z$ to denote a Markov chain formed by the RVs $X, Y, Z$ as defined in [36, Ch. 2.8], and $X \Perp Y$ to denote that $X$ is statistically independent of $Y$.

\section{A. Problem Formulation}

The MARC consists of two transmitters (sources), a receiver (destination) and a relay. Transmitter $i$ has access to the source

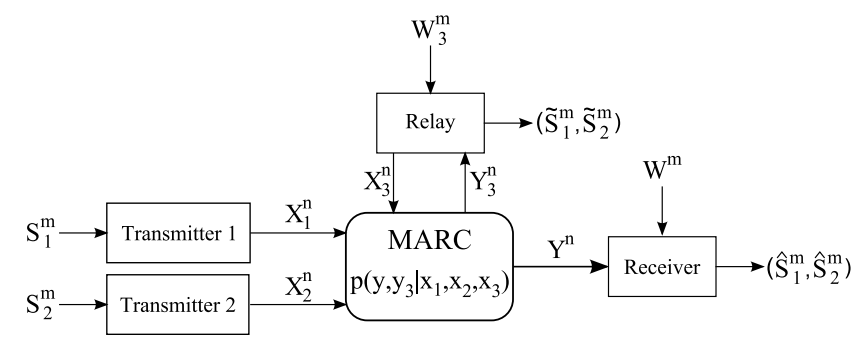

Fig. 1: Multiple-access broadcast relay channel with correlated side information. $\left(\tilde{S}_{1}^{m}, \tilde{S}_{2}^{m}\right)$ are the reconstructions of $\left(S_{1}^{m}, S_{2}^{m}\right)$ at the relay, and $\left(\hat{S}_{1}^{m}, \hat{S}_{2}^{m}\right)$ are the reconstructions at the destination.

sequence $S_{i}^{m}$, for $i=1,2$. The receiver is interested in the lossless reconstruction of the source sequences observed by the two transmitters. The relay has access to side information $W_{3}^{m}$, and the receiver has access to side information $W^{m}$. The objective of the relay is to help the receiver decode the source sequences. For the MABRC the relay is also interested in a lossless reconstruction of the source sequences. Figure 1 depicts the MABRC with side information setup. The MARC is obtained when the reconstruction at the relay is omitted.

The sources and the side information sequences, $\left\{S_{1, k}, S_{2, k}, W_{k}, W_{3, k}\right\}_{k=1}^{m}$, are arbitrarily correlated according to a joint distribution $p\left(s_{1}, s_{2}, w, w_{3}\right)$ over a finite alphabet $\mathcal{S}_{1} \times \mathcal{S}_{2} \times \mathcal{W} \times \mathcal{W}_{3}$, and independent across different sample indices $k$. All nodes know this joint distribution.

For transmission, a discrete memoryless MARC with inputs $X_{1}, X_{2}, X_{3}$ over finite input alphabets $\mathcal{X}_{1}, \mathcal{X}_{2}, \mathcal{X}_{3}$, and outputs $Y, Y_{3}$ over finite output alphabets $\mathcal{Y}, \mathcal{Y}_{3}$, is available. The MARC is memoryless, that is,

$$
\begin{gathered}
p\left(y_{k}, y_{3, k} \mid y^{k-1}, y_{3,1}^{k-1}, x_{1,1}^{k}, x_{2,1}^{k}, x_{3,1}^{k}, s_{1,1}^{m}, s_{2,1}^{m}, w_{3,1}^{m}, w^{m}\right) \\
=p\left(y_{k}, y_{3, k} \mid x_{1, k}, x_{2, k}, x_{3, k}\right), \quad k=1,2, \ldots, n .
\end{gathered}
$$

Definition 1. An $(m, n)$ source-channel code for the MABRC with correlated side information consists of two encoding functions,

$$
f_{i}^{(m, n)}: \mathcal{S}_{i}^{m} \mapsto \mathcal{X}_{i}^{n}, \quad i=1,2,
$$

a set of causal encoding functions at the relay, $\left\{f_{3, k}^{(m, n)}\right\}_{k=1}^{n}$, such that

$$
x_{3, k}=f_{3, k}^{(m, n)}\left(y_{3,1}^{k-1}, w_{3}^{m}\right), \quad 1 \leq k \leq n,
$$

and two decoding functions

$$
\begin{aligned}
& g^{(m, n)}: \mathcal{Y}^{n} \times \mathcal{W}^{m} \mapsto \mathcal{S}_{1}^{m} \times \mathcal{S}_{2}^{m}, \\
& g_{3}^{(m, n)}: \mathcal{Y}_{3}^{n} \times \mathcal{W}_{3}^{m} \mapsto \mathcal{S}_{1}^{m} \times \mathcal{S}_{2}^{m} .
\end{aligned}
$$

An $(m, n)$ source-channel code for the MARC is defined as in Definition 11 with the exception that the decoding function $g_{3}^{(m, n)}$ does not exist.

Definition 2. Let $\hat{S}_{i}^{m}$ denote the reconstruction of $S_{i}^{m}$ at the receiver, and $\tilde{S}_{i}^{m}$ denote the reconstruction of $S_{i}^{m}$ at the relay, for $i=1,2$. The average probability of error, $P_{e}^{(m, n)}$, of an $(m, n)$ code for the MABRC is defined as

$$
\begin{aligned}
P_{e}^{(m, n)} \triangleq \operatorname{Pr}\left\{\left\{\left(\hat{S}_{1}^{m}, \hat{S}_{2}^{m}\right) \neq\left(S_{1}^{m}, S_{2}^{m}\right)\right\}\right. \\
\left.\bigcup\left\{\left(\tilde{S}_{1}^{m}, \tilde{S}_{2}^{m}\right) \neq\left(S_{1}^{m}, S_{2}^{m}\right)\right\}\right\},
\end{aligned}
$$




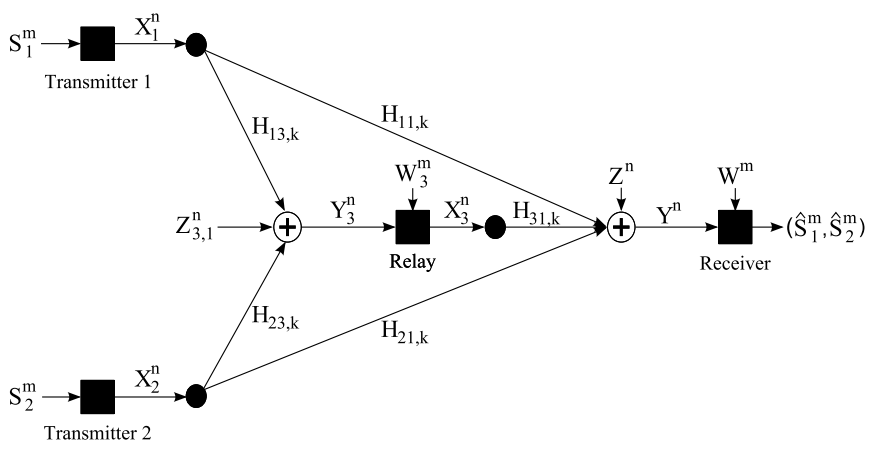

Fig. 2: Sources transmitted over fading Gaussian MARC with side information at the relay and destination.

while for the MARC the average probability of error is defined as

$$
P_{e}^{(m, n)} \triangleq \operatorname{Pr}\left\{\left(\hat{S}_{1}^{m}, \hat{S}_{2}^{m}\right) \neq\left(S_{1}^{m}, S_{2}^{m}\right)\right\} .
$$

Definition 3. A source-channel rate $\kappa$ is said to be achievable for the MABRC if, for every $\epsilon>0$, there exist positive integers $n_{0}, m_{0}$ such that for all $n>n_{0}, m>m_{0}, n \leq \kappa m$ there exists an $(m, n)$ code for which $P_{e}^{(m, n)}<\epsilon$. The same definition applies to the MARC.

\section{B. Fading Gaussian MARCs}

The fading Gaussian MARC is depicted in Figure 2. In fading Gaussian MARCs, the received signals at time $k$ at the receiver and at the relay are given by

$$
\begin{aligned}
Y_{k} & =H_{11, k} X_{1, k}+H_{21, k} X_{2, k}+H_{31, k} X_{3, k}+Z_{k}, \\
Y_{3, k} & =H_{13, k} X_{1, k}+H_{23, k} X_{2, k}+Z_{3, k},
\end{aligned}
$$

for $k=1, \ldots, n$, where $Z$ and $Z_{3}$ are independent of each other, i.i.d., circularly symmetric, complex Gaussian RVs, $\mathcal{C N}(0,1)$. The channel input signals are subject to per-symbol average power constraints: $\mathbb{E}\left\{\left|X_{i}\right|^{2}\right\} \leq P_{i}, i=1,2,3$. In the following it is assumed that the destination knows the instantaneous channel coefficients from the transmitters and the relay to itself, and the relay knows the instantaneous channel coefficients from both transmitters to itself. This is referred to as receiver channel state information (Rx-CSI). Note that the destination does not have CSI on the links arriving at the relay, and that the relay does not have CSI on the links arriving at the destination. It is also assumed that the sources and the relay do not know the channel coefficients on their outgoing links (no transmitter CSI). We represent the CSI at the destination with $\tilde{H}_{1} \triangleq\left(H_{11}, H_{21}, H_{31}\right)$, the CSI at the relay with $\tilde{H}_{3} \triangleq\left(H_{13}, H_{23}\right)$, and define $\tilde{H} \triangleq\left\{H_{11}, H_{21}, H_{31}, H_{13}, H_{23}\right\}$. We consider two types of fading; phase fading and Rayleigh fading:

1) Phase fading channels: The channel coefficients are characterized as $H_{l i, k}=a_{l i} e^{j \Theta_{l i, k}}$, where $a_{l i} \in \mathfrak{R}$ are constants representing the attenuation, and $\Theta_{l i, k}$ are uniformly distributed over $[0,2 \pi)$, i.i.d., and independent of each other and of the additive noises $Z_{3}$ and $Z$.

2) Rayleigh fading channels: The channel coefficients are characterized as $H_{l i, k}=a_{l i} U_{l i, k}$, where $a_{l i} \in \mathfrak{R}$ are constants representing the attenuation, and $U_{l i, k}$ are circularly symmetric, complex Gaussian RVs, $U_{l i, k} \sim$ $\mathcal{C N}(0,1)$, i.i.d., and independent of each other and of the additive noises $Z_{3}$ and $Z$. We define $\tilde{U}=$ $\left\{U_{11}, U_{21}, U_{31}, U_{13}, U_{23}\right\}$.

In both models the values of $a_{l i}$ are fixed and known at all nodes. Observe that the magnitude of the phase-fading process is constant, $\left|H_{l i, k}\right|=a_{l i}$, but for Rayleigh fading the fading magnitude varies between different time instances.

\section{An Achievable Source-Channel Rate BASed on OPERATIONAL SEPARATION}

In this section we derive an achievable source-channel rate for discrete memoryless (DM) MARCs and MABRCs using separate source and channel codes. The achievability is established by using SW source coding, a channel coding scheme similar to the one detailed in [3, Sections II, III], and is based on DF relaying with irregular block Markov encoding, successive decoding at the relay and backward decoding at the destination. The results are summarized in the following theorem:

Theorem 1. For DM MARCs and DM MABRCs with relay and receiver side information as defined in Section II-A, source-channel rate $\kappa$ is achievable if,

$$
\begin{aligned}
& H\left(S_{1} \mid S_{2}, W_{3}\right)<\kappa I\left(X_{1} ; Y_{3} \mid V_{1}, X_{2}, X_{3}\right) \\
& H\left(S_{2} \mid S_{1}, W_{3}\right)<\kappa I\left(X_{2} ; Y_{3} \mid V_{2}, X_{1}, X_{3}\right) \\
& H\left(S_{1}, S_{2} \mid W_{3}\right)<\kappa I\left(X_{1}, X_{2} ; Y_{3} \mid V_{1}, V_{2}, X_{3}\right) \\
& H\left(S_{1} \mid S_{2}, W\right)<\kappa I\left(X_{1}, X_{3} ; Y \mid V_{2}, X_{2}\right) \\
& H\left(S_{2} \mid S_{1}, W\right)<\kappa I\left(X_{2}, X_{3} ; Y \mid V_{1}, X_{1}\right) \\
& H\left(S_{1}, S_{2} \mid W\right)<\kappa I\left(X_{1}, X_{2}, X_{3} ; Y\right),
\end{aligned}
$$

for some joint distribution $p\left(s_{1}, s_{2}, w_{3}, w, v_{1}, v_{2}, x_{1}, x_{2}, x_{3}\right)$ that factorizes as

$$
p\left(s_{1}, s_{2}, w_{3}, w\right) p\left(v_{1}\right) p\left(x_{1} \mid v_{1}\right) p\left(v_{2}\right) p\left(x_{2} \mid v_{2}\right) p\left(x_{3} \mid v_{1}, v_{2}\right) .
$$

Proof: The proof is given in Appendix A

\section{A. Discussion}

Remark 1. In Thm. 11 equations 8a - $8 \mathrm{cc}$ ) are constraints for reliable decoding at the relay, while equations $8 \mathrm{~d}-(8 \mathrm{f})$ are reliable decoding constraints at the destination.

Remark 2. In regular encoding, the codebooks at the sources and at the relay have the same cardinality, see for example [3]. Now, note that the achievable source-channel rate of Thm. 1 is established by using two different Slepian-Wolf coding schemes at different coding rates: one for the relay and one for the destination. The main benefit of different encoding rates is that it allows adapting to the different quality of side information at the relay and destination. Since the rates are different, such encoding cannot be realized with regular encoding and requires an irregular coding scheme for the channel code.

Had we applied regular encoding, it would have led to the merger of some of the constraints in (8), in order to force 
the binning rates to the relay and destination to be equal. For example, $8 \mathrm{a}$ and $(8 \mathrm{~d})$ would be merged into the constraint

$$
\begin{aligned}
\max & \left\{H\left(S_{1} \mid S_{2}, W_{3}\right), H\left(S_{1} \mid S_{2}, W\right)\right\} \\
& <\kappa \min \left\{I\left(X_{1} ; Y_{3} \mid V_{1}, X_{2}, X_{3}\right), I\left(X_{1}, X_{3} ; Y \mid V_{2}, X_{2}\right)\right\} .
\end{aligned}
$$

Hence, regular encoding puts extra constraints on the rates. Accordingly, we conclude that irregular encoding allows higher achievable source-channel rates than regular encoding. When the relay and destination have the same side information ( $W=W_{3}$ ) then the irregular regular encoding schemes achieve the same source-channel rate. This can be observesd by setting $W=W_{3}$ in the above equation, and in (8a and (8d).

Finally, consider regular encoding in the case of a MARC. Here, the relay is not required to recover the source sequences. Therefore, regular encoding requires the merger of the corresponding right-hand sides (RHSs) of the constraints 8a(8f). For example, (8a) and (8d) are merged into the following single constraint

$$
\begin{aligned}
& H\left(S_{1} \mid S_{2}, W\right) \\
& \quad<\kappa \min \left\{I\left(X_{1} ; Y_{3} \mid V_{1}, X_{2}, X_{3}\right), I\left(X_{1}, X_{3} ; Y \mid V_{2}, X_{2}\right)\right\} .
\end{aligned}
$$

This shows that regular encoding is more restrictive than irregular encoding for MARCs as well.

\section{Necessary Conditions on the Achievable SOURCE-CHANNEL RATE FOR DisCRETE MEMORYLESS MARCS AND MABRCS}

In this section we derive necessary conditions for the achievability of a source-channel rate $\kappa$ for MARCs and for MABRCs with correlated sources and side information at the relay and at the destination. The conditions for the MARC are summarized in the following theorem:

Theorem 2. Consider the transmission of arbitrarily correlated sources $S_{1}$ and $S_{2}$ over the DM MARC with relay side information $W_{3}$ and receiver side information $W$. Any achievable source-channel rate $\kappa$ must satisfy the following constraints:

$$
\begin{aligned}
& H\left(S_{1} \mid S_{2}, W\right) \leq \kappa I\left(X_{1}, X_{3} ; Y \mid X_{2}\right) \\
& H\left(S_{2} \mid S_{1}, W\right) \leq \kappa I\left(X_{2}, X_{3} ; Y \mid X_{1}\right) \\
& H\left(S_{1}, S_{2} \mid W\right) \leq \kappa I\left(X_{1}, X_{2}, X_{3} ; Y\right),
\end{aligned}
$$

for some input distribution $p\left(x_{1}, x_{2}, x_{3}\right)$, and the constraints

$$
\begin{aligned}
& H\left(S_{1} \mid S_{2}, W, W_{3}\right) \leq \kappa I\left(X_{1} ; Y, Y_{3} \mid X_{2}, V\right) \\
& H\left(S_{2} \mid S_{1}, W, W_{3}\right) \leq \kappa I\left(X_{2} ; Y, Y_{3} \mid X_{1}, V\right) \\
& H\left(S_{1}, S_{2} \mid W, W_{3}\right) \leq \kappa I\left(X_{1}, X_{2} ; Y, Y_{3} \mid V\right),
\end{aligned}
$$

for some input distribution $p(v)\left(x_{1}, x_{2} \mid v\right) p\left(x_{3} \mid v\right)$, with $|\mathcal{V}| \leq$ 4.

Proof: The proof is given below in Subsection IV-A.

Remark 3. The RHS of the constraints in (11) are similar to the broadcast bound 1 when assuming that all relay information is available at the destination.

\footnotetext{
${ }^{1}$ Here we use the common terminology for the classic relay channel in which the term $I\left(X, X_{1} ; Y\right)$ is referred to as the MAC bound while the term $I\left(X ; Y, Y_{1} \mid X_{1}\right)$ is called the broadcast bound [39. Ch. 16].
}

Remark 4. Setting $\mathcal{X}_{2}=\mathcal{S}_{2}=\phi$, constraints in (10) specialize to the converse of [23, Thm. 3.1] for the relay channel.

Theorem 3. Consider the transmission of arbitrarily correlated sources $S_{1}$ and $S_{2}$ over the DM MABRC with relay side information $W_{3}$ and receiver side information $W$. Any achievable source-channel rate $\kappa$ must satisfy the constraints (10) as well as the following constraints:

$$
\begin{aligned}
& H\left(S_{1} \mid S_{2}, W_{3}\right) \leq \kappa I\left(X_{1} ; Y_{3} \mid X_{2}, X_{3}\right) \\
& H\left(S_{2} \mid S_{1}, W_{3}\right) \leq \kappa I\left(X_{2} ; Y_{3} \mid X_{1}, X_{3}\right) \\
& H\left(S_{1}, S_{2} \mid W_{3}\right) \leq \kappa I\left(X_{1}, X_{2} ; Y_{3} \mid X_{3}\right),
\end{aligned}
$$

for some input distribution $p\left(x_{1}, x_{2}, x_{3}\right)$.

Proof: The proof follows arguments similar to the proof of Thm. 2. and hence, omitted.

\section{A. Proof of Theorem 2}

Let $P_{e}^{(m, n)} \rightarrow 0$ as $n, m \rightarrow \infty$, for a sequence of encoders and decoders $f_{1}^{(m, n)}, f_{2}^{(m, n)}, f_{3}^{(m, n)}, g^{(m, n)}$, such that $\kappa=n / m$ is fixed. By Fano's inequality, [36, Thm. 2.11.1], we have

$$
\begin{aligned}
H\left(S_{1}^{m}, S_{2}^{m} \mid \hat{S}_{1}^{m}, \hat{S}_{2}^{m}\right) & \leq 1+m P_{e}^{(m, n)} \log \left|\mathcal{S}_{1} \times \mathcal{S}_{2}\right| \\
& \triangleq m \delta\left(P_{e}^{(m, n)}\right),
\end{aligned}
$$

where $\delta(x)$ is a non-negative function that approaches $\frac{1}{m}$ as $x \rightarrow 0$. Observe that

$$
\begin{aligned}
H\left(S_{1}^{m}, S_{2}^{m} \mid \hat{S}_{1}^{m}, \hat{S}_{2}^{m}\right) & \stackrel{(a)}{\geq} H\left(S_{1}^{m}, S_{2}^{m} \mid Y^{n}, W^{m}, \hat{S}_{1}^{m}, \hat{S}_{2}^{m}\right) \\
& \stackrel{(b)}{\geq} H\left(S_{1}^{m} \mid Y^{n}, W^{m}, S_{2}^{m}\right),
\end{aligned}
$$

where (a) follows from the fact that conditioning reduces entropy [36, Thm. 2.6.5]; and (b) follows from the fact that $\left(\hat{S}_{1}^{m}, \hat{S}_{2}^{m}\right)$ is a function of $\left(Y^{n}, W^{m}\right)$.

1) Proof of constraints (10): Constraint 10a is a consequence of the following chain of inequalities:

$$
\begin{aligned}
& \sum_{k=1}^{n} I\left(X_{1, k}, X_{3, k} ; Y_{k} \mid X_{2, k}\right) \\
& \quad \stackrel{(a)}{=} \sum_{k=1}^{n}\left[H\left(Y_{k} \mid X_{2, k}\right)-H\left(Y_{k} \mid S_{1}^{m}, S_{2}^{m}, W_{3}^{m}, W^{m},\right.\right. \\
& \left.\left.\qquad X_{1,1}^{k}, X_{2,1}^{k}, X_{3,1}^{k}, Y_{3,1}^{k-1}, Y^{k-1}\right)\right] \\
& \quad \stackrel{(b)}{\geq} \sum_{k=1}^{n}\left[H\left(Y_{k} \mid S_{2}^{m}, W^{m}, Y^{k-1}, X_{2, k}\right)-\right. \\
& \left.\quad H\left(Y_{k} \mid S_{1}^{m}, S_{2}^{m}, W_{3}^{m}, W^{m}, Y^{k-1}\right)\right] \\
& \quad \stackrel{(c)}{=} I\left(S_{1}^{m}, W_{3}^{m} ; Y^{n} \mid S_{2}^{m}, W^{m}\right) \\
& \quad \stackrel{(d)}{\geq} H\left(S_{1}^{m} \mid S_{2}^{m}, W^{m}\right)-H\left(S_{1}^{m} \mid Y^{n}, S_{2}^{m}, W^{m}\right) \\
& \quad \stackrel{(e)}{\geq} m H\left(S_{1} \mid S_{2}, W\right)-m \delta\left(P_{e}^{(m, n)}\right),
\end{aligned}
$$

where (a) follows from the memoryless channel assumption (see (1)) and the Markov relation $\left(S_{1}^{m}, S_{2}^{m}, W_{3}^{m}, W^{m}\right)-$ $\left(X_{1,1}^{k}, X_{2,1}^{k}, X_{3,1}^{k}, Y_{3,1}^{k-1}, Y^{k-1}\right)-Y_{k}$ (see [40]); (b) follows from the fact that conditioning reduces entropy; (c) follows 
from the fact that $X_{2, k}$ is a deterministic function of $S_{2}^{m}$; (d) follows from the non-negativity of the mutual information; and (e) follows from the memoryless sources and side information assumption, and from (13)-14).

Following arguments similar to those that led to (15) we can also show

$$
\begin{aligned}
& \sum_{k=1}^{n} I\left(X_{2, k}, X_{3, k} ; Y_{k} \mid X_{1, k}\right) \\
& \geq m H\left(S_{2} \mid S_{1}, W\right)-m \delta\left(P_{e}^{(m, n)}\right) \\
& \sum_{k=1}^{n} I\left(X_{1, k}, X_{2, k}, X_{3, k} ; Y_{k}\right) \\
& \geq m H\left(S_{1}, S_{2} \mid W\right)-m \delta\left(P_{e}^{(m, n)}\right) .
\end{aligned}
$$

We now recall that the mutual information is concave in the set of joint distributions $p\left(x_{1}, x_{2}, x_{3}\right)$, [36, Thm. 2.7.4]. Thus, taking the limit as $m, n \rightarrow \infty$ and letting $P_{e}^{(m, n)} \rightarrow 0$, 15), (16a) and (16b) result in the constraints in (10).

2) Proof of constraints (11): We begin by defining the following auxiliary RV:

$$
V_{k} \triangleq\left(Y_{3,1}^{k-1}, W_{3}^{m}\right), \quad k=1,2, \ldots, n
$$

Constraint 11a is a consequence of the following chain of inequalities:

$$
\begin{aligned}
& \sum_{k=1}^{n} I\left(X_{1, k} ; Y_{k}, Y_{3, k} \mid X_{2, k}, V_{k}\right) \\
& \stackrel{(a)}{=} \sum_{k=1}^{n}\left[H\left(Y_{k}, Y_{3, k} \mid X_{2, k}, Y_{3,1}^{k-1}, W_{3}^{m}\right)\right. \\
& \left.-H\left(Y_{k}, Y_{3, k} \mid X_{1}^{k}, X_{2}^{k}, X_{3}^{k}, Y_{3,1}^{k-1}, Y^{k-1}, W_{3}^{m}\right)\right] \\
& \stackrel{(b)}{=} \sum_{k=1}^{n}\left[H\left(Y_{k}, Y_{3, k} \mid X_{2, k}, Y_{3,1}^{k-1}, Y^{k-1}, W_{3}^{m}, W^{m}, S_{2}^{m}\right)\right. \\
& -H\left(Y_{k}, Y_{3, k} \mid X_{1}^{k}, X_{2}^{k}, X_{3}^{k}\right. \\
& \left.\left.Y_{3,1}^{k-1}, Y^{k-1}, W_{3}^{m}, W^{m}, S_{1}^{m}, S_{2}^{m}\right)\right] \\
& \stackrel{(c)}{\geq} \sum_{k=1}^{n}\left[H\left(Y_{k}, Y_{3, k} \mid Y_{3,1}^{k-1}, Y^{k-1}, W_{3}^{m}, W^{m}, S_{2}^{m}\right)\right. \\
& \left.-H\left(Y_{k}, Y_{3, k} \mid Y_{3,1}^{k-1}, Y^{k-1}, W_{3}^{m}, W^{m}, S_{1}^{m}, S_{2}^{m}\right)\right] \\
& =I\left(S_{1}^{m} ; Y^{n}, Y_{3}^{n} \mid W_{3}^{m}, W^{m}, S_{2}^{m}\right) \\
& \geq H\left(S_{1}^{m} \mid W_{3}^{m}, W^{m}, S_{2}^{m}\right)-H\left(S_{1}^{m} \mid Y^{n}, W_{3}^{m}, W^{m}, S_{2}^{m}\right) \\
& \stackrel{(d)}{\geq} m H\left(S_{1} \mid S_{2}, W, W_{3}\right)-m \delta\left(P_{e}^{(m, n)}\right) \text {, }
\end{aligned}
$$

where (a) follows from 177, the fact that $X_{3,1}^{k}$ is a deterministic function of $\left(Y_{3,1}^{k-1}, W_{3}^{m}\right)$, and the memoryless channel assumption, (see (10); (b) follows from the fact that conditioning reduces entropy and causality, [40]; (c) follows from the fact that $X_{2, k}$ is a deterministic function of $S_{2}^{m}$, and conditioning reduces entropy; (d) follows again from the fact that conditioning reduces entropy, the memoryless sources and side information assumption, and (13)-114).
Following arguments similar to those that led to (18) we can also show that

$$
\begin{gathered}
\sum_{k=1}^{n} I\left(X_{2, k} ; Y_{k}, Y_{3, k} \mid X_{1, k}, V_{k}\right) \\
\quad \geq m H\left(S_{2} \mid S_{1}, W, W_{3}\right)-m \delta\left(P_{e}^{(m, n)}\right) \\
\sum_{k=1}^{n} I\left(X_{1, k}, X_{2, k} ; Y_{k}, Y_{3, k} \mid V_{k}\right) \\
\quad \geq m H\left(S_{1}, S_{2} \mid W, W_{3}\right)-m \delta\left(P_{e}^{(m, n)}\right) .
\end{gathered}
$$

Next we introduce the time-sharing $\operatorname{RV} Q$, independent of all other RVs, and we have $Q=k$ with probability $1 / n, k \in$ $\{1,2, \ldots, n\}$. We can write

$$
\begin{aligned}
\frac{1}{n} \sum_{k=1}^{n} I\left(X_{1, k}\right. & \left.; Y_{k}, Y_{3, k} \mid X_{2, k}, V_{k}\right) \\
& =I\left(X_{1, Q} ; Y_{Q}, Y_{3, Q} \mid X_{2, Q}, V_{Q}\right) \\
& =I\left(X_{1} ; Y, Y_{3} \mid X_{2}, V\right),
\end{aligned}
$$

where $X_{1} \triangleq X_{1, Q}, X_{2} \triangleq X_{2, Q}, Y \triangleq Y_{Q}, Y_{3} \triangleq Y_{3, Q}$ and $V \triangleq\left(V_{Q}, Q\right)$. Since $\left(X_{1, k}, X_{2, k}\right)$ and $X_{3, k}$ are independent given $V_{k}=\left(Y_{3}^{k-1}, W_{3}^{m}\right)$, for $\bar{v}=(v, k)$ we have

$$
\begin{aligned}
& \operatorname{Pr}\left\{X_{1}=x_{1}, X_{2}=x_{2}, X_{3}=x_{3} \mid V=\bar{v}\right\} \\
& \quad=\operatorname{Pr}\left\{X_{1}=x_{1}, X_{2}=x_{2} \mid V=\bar{v}\right\} \operatorname{Pr}\left\{X_{3}=x_{3} \mid V=\bar{v}\right\} .
\end{aligned}
$$

Hence, the probability distribution is of the form given in $\mathrm{Thm}$. 2 for the constraints in (11). Finally, repeating the steps leading to (20) for (19a) and (19b), and taking the limit $m, n \rightarrow \infty$, leads to the constraints in 11 .

\section{Optimality of Source-Channel Separation For FADING GAUSSIAN MARCS AND MABRCS}

In this section we study source-channel coding for fading Gaussian MARCs and MABRCs. We derive conditions for the optimality of source-channel separation for the phase and Rayleigh fading models. We begin by considering phase fading Gaussian MARCs, defined in (7). The result is stated in the following theorem:

Theorem 4. Consider the transmission of arbitrarily correlated sources $S_{1}$ and $S_{2}$ over a phase fading Gaussian MARC with receiver side information $W$ and relay side information $W_{3}$. Let the channel inputs be subject to per-symbol power constraints specified by

$$
\mathbb{E}\left\{\left|X_{i}\right|^{2}\right\} \leq P_{i}, \quad i=1,2,3,
$$

and the channel coefficients and power constraints $\left\{P_{i}\right\}_{i=1}^{3}$ satisfy

$$
\begin{aligned}
a_{11}^{2} P_{1}+a_{31}^{2} P_{3} & \leq a_{13}^{2} P_{1} \\
a_{21}^{2} P_{2}+a_{31}^{2} P_{3} & \leq a_{23}^{2} P_{2} \\
a_{11}^{2} P_{1}+a_{21}^{2} P_{2}+a_{31}^{2} P_{3} & \leq a_{13}^{2} P_{1}+a_{23}^{2} P_{2} .
\end{aligned}
$$

A source-channel rate $\kappa$ is achievable if

$$
\begin{aligned}
& H\left(S_{1} \mid S_{2}, W\right)<\kappa \log _{2}\left(1+a_{11}^{2} P_{1}+a_{31}^{2} P_{3}\right) \\
& H\left(S_{2} \mid S_{1}, W\right)<\kappa \log _{2}\left(1+a_{21}^{2} P_{2}+a_{31}^{2} P_{3}\right) \\
& H\left(S_{1}, S_{2} \mid W\right)<\kappa \log _{2}\left(1+a_{11}^{2} P_{1}+a_{21}^{2} P_{2}+a_{31}^{2} P_{3}\right) .
\end{aligned}
$$


Conversely, if source-channel rate $\kappa$ is achievable, then conditions (24) are satisfied with $<$ replaced by $\leq$.

Proof: The necessity part is proved in Subsection V-A1 and sufficiency is shown in subsection $\mathrm{V}-\mathrm{A} 2$.

Remark 5. To achieve the source-channel rates $\kappa$ stated in Thm. 4 we use channel inputs distributed according to $X_{i} \sim \mathcal{C} \mathcal{N}\left(0, P_{i}\right), i \in\{1,2,3\}$, all mutually independent, and generate the codebooks in an i.i.d. manner. The relay employs the DF scheme.

Remark 6. Note that the phase fading MARC is not degraded in the sense of [11], see also [2, Remark 33].

Remark 7. The result of Thm. 4 relies on the assumptions of additive Gaussian noise, Rx-CSI, and i.i.d. fading coefficients such that the phases of the fading coefficients are mutually independent, uniformly distributed, and independent of their magnitudes. These assumptions are essential for the result.

Remark 8. Observe that from the achievability result of [31. Appendix A], it follows that the optimal source code and channel code used in the proof of Thm. 4 are separate and stand-alone. Thus, informational separation is optimal. We now provide an intuitive explanation for the optimality of separation for the current scenario: Note that when separate and stand-alone source and channel codes are used, the channel inputs of the two transmitters, $X_{1}$ and $X_{2}$, are be mutually independent, i.e., $p\left(x_{1}, x_{2}\right)=p\left(x_{1}\right) p\left(x_{2}\right)$. This puts a restriction on the feasible joint distributions for generating the channel codebooks. Using a joint source-channel code allows generating channel inputs that are statically dependent on the source symbols. Since $S_{1}$ and $S_{2}$ are correlated this induces statistical dependence between the channel inputs $X_{1}$ and $X_{2}$. This, in turn, enlarges the set of feasible joint input distributions which can be realized for generating the channel codebooks; and therefore, the set of achievable transmission rates over the channel may increase. However, for fading Gaussian MARCs, due to the uniformly distributed phases of the channel coefficients, in the absence of Tx-CSI, the received signal components (from the sources and from the relay) at the destination are uncorrelated. Therefore, there is no advantage, from the perspective of channel coding, in generating correlated channel inputs. Coupled with the entropy maximization property of the Gaussian RVs, we conclude that the optimal channel inputs are mutually independent. From this discussion it follows that there is no benefit from joint sourcechannel coding, and source-channel separation is optimal.

Remark 9. There exist examples of channels which are not fading Gaussian channels, but satisfy the rest of the assumptions detailed in Section II-B for which the DF-based sufficient conditions of Thm. 1 are not optimal. One such example is the Gaussian relay channel with fixed channel coefficients, see also discussion in [2, Section VII-B].

Next, we consider source transmission over Rayleigh fading MARCs.

Theorem 5. Consider transmission of arbitrarily correlated sources $S_{1}$ and $S_{2}$ over a Rayleigh fading Gaussian MARC with receiver side information $W$ and relay side information $W_{3}$. Let the channel inputs be subject to per-symbol power constraints as in (22), and let the channel coefficients and the power constraints $\left\{P_{i}\right\}_{i=1}^{3}$ satisfy

$$
\begin{array}{r}
1+a_{11}^{2} P_{1}+a_{31}^{2} P_{3} \leq \frac{a_{13}^{2} P_{1}}{e^{\frac{1}{a_{13}^{2} P_{1}}} E_{1}\left(\frac{1}{a_{13}^{2} P_{1}}\right)} \\
1+a_{21}^{2} P_{2}+a_{31}^{2} P_{3} \leq \frac{a_{23}^{2} P_{2}}{e^{\frac{1}{a_{23}^{2} P_{2}}} E_{1}\left(\frac{1}{a_{23}^{2} P_{2}}\right)} \\
1+a_{11}^{2} P_{1}+a_{21}^{2} P_{2}+a_{31}^{2} P_{3} \leq \\
\frac{a_{23}^{2} P_{2}-a_{13}^{2} P_{1}}{\left(e^{\frac{1}{a_{23}^{2} P_{2}}} E_{1}\left(\frac{1}{a_{23}^{2} P_{2}}\right)-e^{\frac{1}{a_{13}^{2} P_{1}}} E_{1}\left(\frac{1}{a_{13}^{2} P_{1}}\right)\right)},
\end{array}
$$

where $E_{1}(x) \triangleq \int_{q=x}^{\infty} \frac{1}{q} e^{-q} d q$, see [42, Eqn. (5.1.1)]. A sourcechannel rate $\kappa$ is achievable if

$$
\begin{gathered}
H\left(S_{1} \mid S_{2}, W\right)<\kappa \mathbb{E}_{\tilde{U}}\left\{\operatorname { l o g } _ { 2 } \left(1+a_{11}^{2}\left|U_{11}\right|^{2} P_{1}\right.\right. \\
\left.\left.+a_{31}^{2}\left|U_{31}\right|^{2} P_{3}\right)\right\} \\
H\left(S_{2} \mid S_{1}, W\right)<\kappa \mathbb{E}_{\tilde{U}}\left\{\operatorname { l o g } _ { 2 } \left(1+a_{21}^{2}\left|U_{21}\right|^{2} P_{2}\right.\right. \\
\left.\left.+a_{31}^{2}\left|U_{31}\right|^{2} P_{3}\right)\right\} \\
H\left(S_{1}, S_{2} \mid W\right)<\kappa \mathbb{E}_{\tilde{U}}\left\{\operatorname { l o g } _ { 2 } \left(1+a_{11}^{2}\left|U_{11}\right|^{2} P_{1}+\right.\right. \\
\left.\left.a_{21}^{2}\left|U_{21}\right|^{2} P_{2}+a_{31}^{2}\left|U_{31}\right|^{2} P_{3}\right)\right\}
\end{gathered}
$$

Conversely, if source-channel rate $\kappa$ is achievable, then conditions (26) are satisfied with $<$ replaced by $\leq$.

Proof: The proof uses [31, Corollary 1] and follows similar arguments to those in the proof of Thm. 4.

Remark 10. The source-channel rate $\kappa$ in Thm. 5 is achieved by using $X_{i} \sim \mathcal{C N}\left(0, P_{i}\right), i \in\{1,2,3\}$, all i.i.d. and independent of each other, and applying DF at the relay.

\section{A. Proof of Theorem 4}

1) Necessity Proof of Theorem 4 . Consider the necessary conditions of Thm. 2. We first note that the phase fading MARC model specified in Section II-B exactly belongs to the class of fading relay channels with Rx-CSI stated in [2, Thm. 8]. Thus, from [2, Thm. 8] it follows that for phase fading MARCs with Rx-CSI, the mutual information expressions on the RHS of (10) are simultaneously maximized by $X_{1}, X_{2}, X_{3}$ mutually independent, zero-mean complex Gaussian RVs, $X_{i} \sim \mathcal{C N}\left(0, P_{i}\right), i=1,2,3$. Applying this input p.d.f. to (10) yields the expressions in (24). Therefore, for phase fading MARCs, when conditions (23) hold, the conditions in (24) coincide with the necessary conditions of Thm. 2] after replacing " $<$ " with " $\leq$ ".

2) Sufficiency Proof of Theorem 4 .

- Codebook construction: For $i=1,2$, assign every $\mathbf{s}_{i} \in \mathcal{S}_{i}^{m}$ to one of $2^{m R_{i}}$ bins independently according to a uniform distribution over $\mathcal{U}_{i} \triangleq\left\{1,2, \ldots, 2^{m R_{i}}\right\}$. Denote these assignments by $f_{i}$. Set $\hat{R}_{i}=\frac{1}{\kappa} R_{i}, i=1,2$, and let $X_{k} \sim \mathcal{C N}\left(0, P_{k}\right), \quad k=1,2,3$, all mutually independent.

\footnotetext{
${ }^{2} \mathrm{Rx}$-CSI is incorporated into Thm. 2 by replacing $Y$ with $\left(Y, \tilde{H}_{1}\right)$ in Eqns (10), and $\left(Y, Y_{3}\right)$ with $\left(Y, Y_{3}, \tilde{H}\right)$ in Eqns. (11), and then by using the fact that due to the absence of Tx-CSI, $\left(\tilde{H}_{1}, \tilde{H}\right) \Perp\left(X_{1}, X_{2}, X_{3}\right)$, see [2] Eq. (50)].
} 
Construct a channel code based on DF with rates $\hat{R}_{1}$ and $\hat{R}_{2}$, and with blocklength $n$, as detailed in [31, Appendix A].

- Encoding: Consider sequences of length $B m, s_{i}^{B m} \in$ $\mathcal{S}_{i}^{B m}, i=1,2, w^{B m} \in \mathcal{W}^{B m}$. Partition each sequence into $B$ length- $m$ subsequences, $\mathbf{s}_{i, b}, i=1,2$, and $\mathbf{w}_{b}, b=1,2, \ldots, B$. A total of $B m$ source samples are transmitted over $B+1$ blocks of $n$ channel symbols each. Setting $n=\kappa m$, and increasing $B$ we obtain a source-channel rate $(B+1) n / B m \rightarrow$ $n / m=\kappa$ as $B \rightarrow \infty$.

At block $b, b=1,2, \ldots, B$, source terminal $i, i=1,2$, observes $\mathbf{s}_{i, b}$ and finds its corresponding bin index $u_{i, b} \in \mathcal{U}_{i}$. Each transmitter sends its corresponding bin index using the channel code described in [31, Appendix A]. Assume that at time $b$ the relay knows $\left(u_{1, b-1}, u_{2, b-1}\right)$. The relay sends these bin indices using the encoding scheme described in [31, Appendix A].

- Decoding and error probability analysis: We apply the decoding rule of [31, Eqn. (A2)]. From the error probability analysis in [31, Appendix A], it follows that, when the channel coefficients and the channel input power constraints satisfy the conditions in 23, the RHSs of the constraints in (24) characterize the ergodic capacity region (in the sense of [2, Eq. (51)]) of the phase fading Gaussian MARC (see [2, Thm. 9], [31, Appendix A]). Hence, when consitions (23) are satisfied, the transmitted bin indices $\left\{u_{1, b}, u_{2, b}\right\}_{b=1}^{B}$ can be reliably decoded at the destination as long as

$$
\begin{aligned}
R_{1}<\kappa \log _{2}\left(1+a_{11}^{2} P_{1}+a_{31}^{2} P_{3}\right) \\
R_{2}<\kappa \log _{2}\left(1+a_{21}^{2} P_{2}+a_{31}^{2} P_{3}\right) \\
R_{1}+R_{2}<\kappa \log _{2}\left(1+a_{11}^{2} P_{1}+a_{21}^{2} P_{2}+a_{31}^{2} P_{3}\right) .
\end{aligned}
$$

Decoding the sources at the destination: The decoded bin indices, denoted $\left(\tilde{u}_{1, b}, \tilde{u}_{2, b}\right), b=1,2, \ldots, B$, are given to the source decoder at the destination. Using the bin indices $\left(\tilde{u}_{1, b}, \tilde{u}_{2, b}\right)$ and the side information $\mathbf{w}_{b}$, the source decoder at the destination estimates $\left(\mathbf{s}_{1, b}, \mathbf{s}_{2, b}\right)$ by looking for a unique pair of sequences $\left(\tilde{\mathbf{s}}_{1}, \tilde{\mathbf{s}}_{2}\right) \in S_{1}^{m} \times S_{2}^{m}$ that satisfies $f_{1}\left(\tilde{\mathbf{s}}_{1}\right)=$ $\tilde{u}_{1, b}, f_{2}\left(\tilde{\mathbf{s}}_{2}\right)=\tilde{u}_{2, b}$ and $\left(\tilde{\mathbf{s}}_{1}, \tilde{\mathbf{s}}_{2}, \mathbf{w}_{b}\right) \in A_{\epsilon}^{*(m)}\left(S_{1}, S_{2}, W\right)$. From the Slepian-Wolf theorem [36, Thm 14.4.1], $\left(\mathbf{s}_{1, b}, \mathbf{s}_{2, b}\right)$ can be reliably decoded at the destination if

$$
\begin{aligned}
& H\left(S_{1} \mid S_{2}, W\right) \leq R_{1} \\
& H\left(S_{2} \mid S_{1}, W\right) \leq R_{2} \\
& H\left(S_{1}, S_{2} \mid W\right) \leq R_{1}+R_{2} .
\end{aligned}
$$

Combining conditions (27) and (28) yields (24), and completes the achievability proof.

Remark 11. Note that in the sufficiency proof in Section $\mathrm{V}$-A2 we used the code construction and the decoding procedure of [31, Appendix A], which are designed specifically for fading MARCs. The reason we did not use the result of Thm. 1 is that for the channel inputs to be mutually independent, we must set $V_{1}=V_{2}=\phi$ in Thm. 11. But, with such an assignment, the decoding rule of the channel code at the destination given by Eqn. A.2 does not apply, as this rule decodes the information carried by the auxiliary $R V s$. For the same reason we did not simply cite [2, Thm. 9] for the channel coding part of the sufficiency proof of Thm. 4. We conclude that a specialized channel code must be constructed for fading channels. The issue of channel coding for fading MARCs has already been addressed in [31], and we refer to [31] for a detailed discussion.

\section{B. Fading MABRCs}

Optimality of informational separation can also be established for MABRCs by using the results for MARCs with three additional constraints. The result is stated in the following theorem:

Theorem 6. For phase fading MABRCs for which the conditions in 23) hold together with

$$
\begin{aligned}
& H\left(S_{1} \mid S_{2}, W_{3}\right) \leq H\left(S_{1} \mid S_{2}, W\right) \\
& H\left(S_{2} \mid S_{1}, W_{3}\right) \leq H\left(S_{2} \mid S_{1}, W\right) \\
& H\left(S_{1}, S_{2} \mid W_{3}\right) \leq H\left(S_{1}, S_{2} \mid W\right),
\end{aligned}
$$

a source-channel rate $\kappa$ is achievable if conditions (24) are satisfied. Conversely, if a source-channel rate $\kappa$ is achievable, then conditions (24) are satisfied with $<$ replaced by $\leq$. The same statement holds for Rayleigh fading MABRCs with 25) replacing (23) and (26) replacing (24).

Proof: The sufficiency proof of Thm. 6 differs from the sufficiency proof of Thm. 4 only due to decoding requirement of the source sequences at the relay. Conditions (23) imply that reliable decoding of the channel code at the destination implies reliable decoding of the channel code at the relay. Conditions (29) imply that the relay achievable source rate region contains the destination achievable source rate region, and therefore, reliable decoding of the source code at the destination implies reliable decoding of the source code at the relay. Hence, if conditions (23), (24), and (29) hold, $\left(\mathbf{s}_{1, b}, \mathbf{s}_{2, b}\right), b=1,2, \ldots, B$, can be reliably decoded at both the relay and the destination. Necessity of (24) follows from the necessary conditions of Thm. 3. and by following similar arguments to the necessity proof of Thm. 4 .

The extension to Rayleigh fading is similar to the one done for MARCs (from Thm. 4 to Thm. 5).

Remark 12. Conditions (29) imply that for the scenario described in Thm. 4 regular and irregular encoding yield the same source-channel achievable rates (see Remark 2); hence, the channel code construction of [31, Appendix A] can be used without any rate loss.

\section{Joint Source-Channel ACHIEVABle RATES FOR DisCRETE MEMORYLESS MARCS AND MABRCS}

In this section we derive two sets of sufficient conditions for the achievability of source-channel rate $\kappa=1$ for DM MARCs and MABRCs with correlated sources and side information. Both achievability schemes are established by using a combination of SW source coding, the CPM technique, and a DF scheme with successive decoding at the relay and backward decoding at the destination. The techniques differ in the way the source codes are combined. In the first scheme (Thm.7), SW source coding is used for encoding information to the destination and CPM is used for encoding information to the relay. In the second scheme (Thm. 8), CPM is used 
for encoding information to the destination while SW source coding is used for encoding information to the relay.

Before presenting the results, we first motivate this section by recalling that separate source-channel coding is sub-optimal for the MAC [7]. This implies that in general, separate sourcechannel coding is sub-optimal for the MARC and MABRC as well.

\section{A. Joint Source-Channel Coding for MARCs and MABRCs}

Thm. 7 and Thm. 8 below present two new sets of sufficient conditions for the achievability of source-channel rate $\kappa=1$, obtained by combining SW source coding and CPM. For the sources $S_{1}$ and $S_{2}$ we define common information in the sense of Gács, Körner [44] and Witsenhausen [45], as $T \triangleq h_{1}\left(S_{1}\right)=$ $h_{2}\left(S_{2}\right)$, where $h_{1}$ and $h_{2}$ are deterministic functions. We now state the theorems:

Theorem 7. For DM MARCs and MABRCs with relay and receiver side information as defined in Section $\amalg-\mathrm{A}$ and source pair $\left(S_{1}, S_{2}\right)$ with common part $T \triangleq h_{1}\left(S_{1}\right)=h_{2}\left(S_{2}\right)$, a source-channel rate $\kappa=1$ is achievable if,

$$
\begin{aligned}
H\left(S_{1} \mid S_{2}, W_{3}\right) & <I\left(X_{1} ; Y_{3} \mid S_{2}, V_{1}, X_{2}, X_{3}, W_{3}, Q\right) \\
H\left(S_{2} \mid S_{1}, W_{3}\right) & <I\left(X_{2} ; Y_{3} \mid S_{1}, V_{2}, X_{1}, X_{3}, W_{3}, Q\right) \\
H\left(S_{1}, S_{2} \mid W_{3}, T\right) & <I\left(X_{1}, X_{2} ; Y_{3} \mid V_{1}, V_{2}, X_{3}, W_{3}, T, Q\right) \\
H\left(S_{1}, S_{2} \mid W_{3}\right) & <I\left(X_{1}, X_{2} ; Y_{3} \mid V_{1}, V_{2}, X_{3}, W_{3}\right) \\
H\left(S_{1} \mid S_{2}, W\right) & <I\left(X_{1}, X_{3} ; Y \mid S_{1}, V_{2}, X_{2}, Q\right) \\
H\left(S_{2} \mid S_{1}, W\right) & <I\left(X_{2}, X_{3} ; Y \mid S_{2}, V_{1}, X_{1}, Q\right) \\
H\left(S_{1}, S_{2} \mid W\right) & <I\left(X_{1}, X_{2}, X_{3} ; Y \mid S_{1}, S_{2}, Q\right),
\end{aligned}
$$

for some joint distribution that factorizes as

$$
\begin{aligned}
& p\left(s_{1}, s_{2}, w_{3}, w\right) p(q) p\left(v_{1}\right) p\left(x_{1} \mid s_{1}, v_{1}, q\right) \times \\
& \quad p\left(v_{2}\right) p\left(x_{2} \mid s_{2}, v_{2}, q\right) p\left(x_{3} \mid v_{1}, v_{2}\right) p\left(y_{3}, y \mid x_{1}, x_{2}, x_{3}\right) .
\end{aligned}
$$

Proof: The proof is given in Appendix B]

Theorem 8. For DM MARCs and MABRCs with relay and receiver side information as defined in Section $\amalg-\mathrm{A}$ and source pair $\left(S_{1}, S_{2}\right)$ with common part $T \triangleq h_{1}\left(S_{1}\right)=h_{2}\left(S_{2}\right)$, a source-channel rate $\kappa=1$ is achievable if,

$$
\begin{gathered}
H\left(S_{1} \mid S_{2}, W_{3}\right)<I\left(X_{1} ; Y_{3} \mid S_{1}, X_{2}, X_{3}, Q\right) \\
H\left(S_{2} \mid S_{1}, W_{3}\right)<I\left(X_{2} ; Y_{3} \mid S_{2}, X_{1}, X_{3}, Q\right) \\
H\left(S_{1}, S_{2} \mid W_{3}\right)<I\left(X_{1}, X_{2} ; Y_{3} \mid S_{1}, S_{2}, X_{3}, Q\right) \\
H\left(S_{1} \mid S_{2}, W\right)<I\left(X_{1}, X_{3} ; Y \mid S_{2}, X_{2}, W, Q\right) \\
H\left(S_{2} \mid S_{1}, W\right)<I\left(X_{2}, X_{3} ; Y \mid S_{1}, X_{1}, W, Q\right) \\
H\left(S_{1}, S_{2} \mid W, T\right)<I\left(X_{1}, X_{2}, X_{3} ; Y \mid W, T, Q\right) \\
H\left(S_{1}, S_{2} \mid W\right)<I\left(X_{1}, X_{2}, X_{3} ; Y \mid W\right)
\end{gathered}
$$

for some joint distribution that factorizes as

$$
\begin{aligned}
& p\left(s_{1}, s_{2}, w_{3}, w\right) p(q) p\left(x_{1} \mid s_{1}, q\right) \times \\
& p\left(x_{2} \mid s_{2}, q\right) p\left(x_{3} \mid s_{1}, s_{2}, q\right) p\left(y_{3}, y \mid x_{1}, x_{2}, x_{3}\right) .
\end{aligned}
$$

Proof: The proof is given in Appendix C

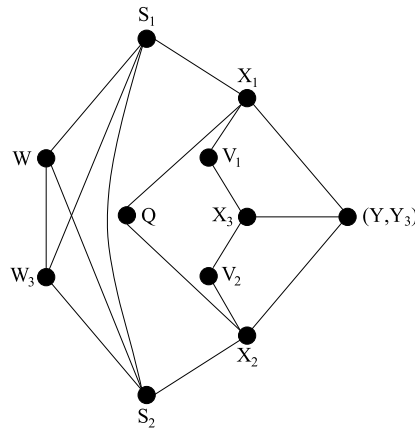

(a)

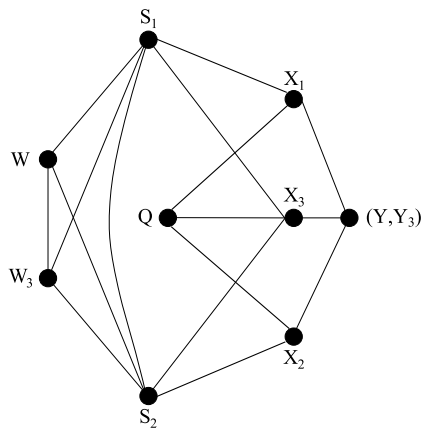

(b)
Fig. 3: (a) Diagram of the Markov chain for the joint distribution considered in 31; (b) Diagram of the Markov chain for the joint distribution considered in 33 .

\section{B. Discussion}

Figure 3 illustrates the Markov chains for the joint distributions considered in Thm. 7 and Thm. 8

Remark 13. Conditions 30a-30d in Thm. 7 and conditions (32a) - 32c) in Thm. 8 are constraints for decoding at the relay, while conditions $30 \mathrm{e}-(30 \mathrm{~g})$ and $32 \mathrm{~d}-32 \mathrm{~g}$ ) are decoding constraints at the destination.

Remark 14. Each mutual information expression on the RHS of the constraints in Thm. 7 and Thm. 8 represents the rate of one of two encoding types: either source-channel encoding via CPM or SW encoding. Consider Thm. 7 Here, $V_{1}$ and $V_{2}$ represent the binning information for $S_{1}$ and $S_{2}$, respectively. Observe that the left-hand side (LHS) of condition (30a) is the entropy of $S_{1}$ when $\left(S_{2}, W_{3}\right)$ are known. On the RHS of 30a), as $S_{2}, V_{1}, X_{2}, X_{3}, W_{3}$ and $Q$ are given, the mutual information expression $I\left(X_{1} ; Y_{3} \mid S_{2}, V_{1}, X_{2}, X_{3}, W_{3}, Q\right)$ represents the available rate that can be used for encoding information on the source $S_{1}$, in excess of the bin index represented by $V_{1}$. The LHS of condition (30) is the entropy of $S_{1}$ when $\left(S_{2}, W\right)$ are known. The RHS of condition (30e) expresses the amount of binning information that can be reliably transmitted cooperatively by transmitter 1 and the relay to the destination. This can be seen by rewriting the mutual information expression in 30e as $I\left(X_{1}, X_{3} ; Y \mid S_{1}, V_{2}, X_{2}, Q\right)=$ $I\left(X_{1}, X_{3} ; Y \mid S_{1}, S_{2}, X_{2}, V_{2}, W, Q\right)$. As $S_{1}$ is given, this expression represents the rate at which the bin index of source $S_{1}$ can be transmitted to the destination in excess of the source-channel rate for encoding $S_{1}$ (see Appendix B). Therefore, each mutual information expression in 30a and (30e) represents different types of information sent by the source: either source-channel codeword to the relay as in 30a ; or bin index to the destination as in (30e). This difference is because SW source coding is used for encoding information to the destination while CPM is used for encoding information to the relay.

Similarly, consider the RHS of 32a in Thm. 8. The mutual information expression $I\left(X_{1} ; Y_{3} \mid S_{1}, X_{2}, X_{3}, Q\right)=$ $I\left(X_{1} ; Y_{3} \mid S_{1}, S_{2}, X_{2}, X_{3}, W_{3}, Q\right)$ represents the rate that can be used for encoding the bin index of source $S_{1}$ to the relay (see Appendix [C), since $S_{1}$ is given. In contrast, the 
mutual information expression $I\left(X_{1}, X_{3} ; Y \mid S_{2}, X_{2}, W, Q\right)$ on the RHS of (32d) represents the available rate that can be used for cooperative source-channel encoding of the source $S_{1}$ to the destination. This follows as $S_{2}, X_{2}, W$ and $Q$ are given.

Remark 15. Thm. 7 and Thm. 8 establish different sufficient conditions. In [7] it was shown that separate source and channel coding is generally suboptimal for transmitting correlated sources over MACs. It then directly follows that separate coding is also suboptimal for DM MARCs and MABRCs. In Thm. 7 the CPM technique is used for encoding information to the relay, while in Thm. $8 \mathrm{SW}$ coding concatenated with independent channel coding is used for encoding information to the relay. Coupled with the above observation, this implies that the relay decoding constraints of $\mathrm{Thm}$. 7 are generally looser compared to the relay decoding constraints of Thm. 8 Using similar reasoning we conclude that the destination decoding constraints of Thm. 8 are looser compared to the destination decoding constraints of Thm. 7 (as long as coordination is possible, see Remark 18). Considering the distribution chains in (31) and (33) we conclude that these two theorems represent different sets of sufficient conditions, and neither are special cases of each other nor include one another.

Remark 16. Thm. 7 coincides with Thm. 1 for $\kappa=1$ and no common information: Consider the case in which the source pair $\left(S_{1}, S_{2}\right)$ has no common part, that is $\mathcal{T}=\phi$, and let $\mathcal{Q}=\phi$ as well. For an input distribution

$$
\begin{aligned}
& p\left(s_{1}, s_{2}, w_{3}, w, v_{1}, v_{2}, x_{1}, x_{2}, x_{3}\right) \\
& \quad=p\left(s_{1}, s_{2}, w_{3}, w\right) p\left(v_{1}\right) p\left(x_{1} \mid v_{1}\right) p\left(v_{2}\right) p\left(x_{2} \mid v_{2}\right) p\left(x_{3} \mid v_{1}, v_{2}\right),
\end{aligned}
$$

conditions (30) specialize to conditions (8), and the transmission scheme of Thm. 7 (see Appendix B) specializes to a separation-based achievability scheme of Thm. 1 for $\kappa=1$, under these assumptions.

Remark 17. In both Thm. 7 and Thm. 8 the conditions stemming from the CPM technique can be specialized to the sufficient conditions of [7, Thm. 1] derived for a MAC. In Thm. 7, letting $\mathcal{V}_{1}=\mathcal{V}_{2}=\mathcal{X}_{3}=\mathcal{W}_{3}=\phi$, specializes the relay conditions in (30a)-(30d) to the ones in [7, Thm. 1] with $Y_{3}$ as the destination. In Thm. 8, letting $\mathcal{X}_{3}=\mathcal{W}=\phi$, specializes the destination conditions in $(32 \mathrm{~d})-(32 \mathrm{~g})$ to the ones in [7, Thm. 1] with $Y$ as the destination.

Remark 18. Thm. 7 is optimal in some scenarios: consider the cooperative relay-broadcast channel (CRBC) depicted in Figure 4. This model is a special case of a MARC obtained when there is a single source terminal. For the CRBC with correlated relay and destination side information, we can identify exactly the optimal source-channel rate using Thm. 1 and Thm. 3 This result was previously obtained in [23]:

Corollary. ([23, Thm. 3.1]) For the CRBC with relay and receiver side information, source-channel rate $\kappa$ is achievable if

$$
\begin{aligned}
H\left(S_{1} \mid W_{3}\right) & <\kappa I\left(X_{1} ; Y_{3} \mid X_{3}\right) \\
H\left(S_{1} \mid W\right) & <\kappa I\left(X_{1}, X_{3} ; Y\right),
\end{aligned}
$$

for some input distribution $p\left(s_{1}, w_{3}, w\right) p\left(x_{1}, x_{3}\right)$. Conversely, if rate $\kappa$ is achievable then the conditions in (34) are sat-

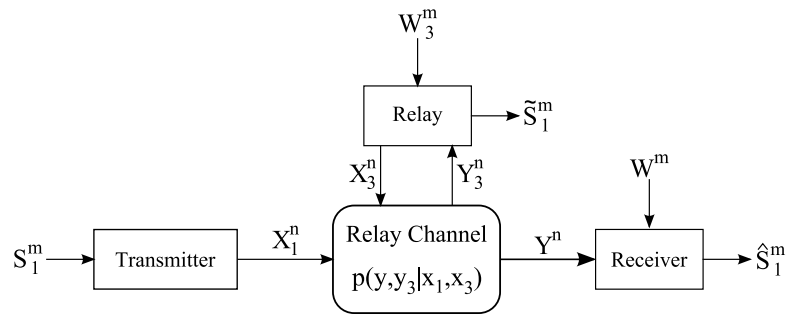

Fig. 4: The cooperative relay-broadcast channel with correlated side information. $\tilde{S}_{1}^{m}$ and $\hat{S}_{1}^{m}$ are the estimates of $S_{1}^{m}$ at the relay and destination, respectively.

isfied with $<$ replaced by $\leq$ for some input distribution $p\left(s_{1}, w_{3}, w\right) p\left(x_{1}, x_{3}\right)$.

Proof: The achievability follows from Thm. 1 by assigning $X_{3}=V_{1}$ and $\mathcal{S}_{2}=\mathcal{X}_{2}=\mathcal{V}_{2}=\phi$. The converse follows from Thm. 3 .

For source-channel rate $\kappa=1$, the conditions in (34) can also be obtained from Thm. 7 by letting $V_{1}=X_{3}$, $\mathcal{S}_{2}=\mathcal{X}_{2}=\mathcal{V}_{2}=\mathcal{T}=\mathcal{Q}=\phi$ and considering an input distribution independent of the sources. Observe that Thm. 8 is not optimal for the CRBC: consider the conditions in Thm. 8 with $\mathcal{S}_{2}=\mathcal{X}_{2}=\mathcal{T}=\mathcal{Q}=\phi$. For this assignment we obtain the following sufficient conditions:

$$
\begin{aligned}
H\left(S_{1} \mid W_{3}\right) & <I\left(X_{1} ; Y_{3} \mid X_{3}, S_{1}\right) \\
H\left(S_{1} \mid W\right) & <I\left(X_{1}, X_{3} ; Y \mid W\right),
\end{aligned}
$$

for some input distribution that factorizes as

$$
p\left(s_{1}, w_{3}, w\right) p\left(x_{1} \mid s_{1}\right) p\left(x_{3} \mid s_{1}\right) .
$$

Note that the RHSs of (35a) and (35b) are smaller than or equal to the RHSs in (34a) and (34b), respectively. Moreover, not all joint input distributions that are feasible for [23, Thm. 3.1] are also feasible with (35c). Hence, the conditions obtained from Thm. 8 for the CRBC setup with $\kappa=1$ are stricter than those obtained from Thm. 7, further illustrating the fact that the two sets of sufficient conditions are not equivalent. We conclude that the downside of using CPM to the destination as applied in this work is that it places constraints on the distribution chain, thereby constraining the achievable coordination between the sources and the relay. Due to this restriction, when there is only a single source, the joint distributions of the source and the relay $\left(X_{1}\right.$ and $\left.X_{3}\right)$ permitted for the scheme of Thm. 8 do not exhaust the entire space of joint distributions, and as a result, the source-channel sufficient conditions obtained from Thm. 8 are generally more restrictive than those obtained from Thm. 7 for the single source case. However, in the case of a MARC it is not possible to determine whether either of the schemes is universally better than the other.

Remark 19. Note that in Thm. 7, it is not useful to generate the relay channel input statistically dependent on the common information, that is, on the auxiliary RV $Q$. To understand why, recall that in Thm. $7 \mathrm{SW}$ source coding is used for encoding information to the destination, while CPM is used for encoding information to the relay. The optimality of SW encoding [12] implies that letting the decoder know the common information 
will not improve the constraints for the source decoder at the destination, as these are based on the SW decoder (see (B.46)). Moreover, note that even though CPM is used for encoding information to the relay, sending common information via the relay channel input will not improve the decoding constraints at the relay. This follows from the fact that in the DF scheme cooperation information is used with a delay of one block. Therefore, common information at the relay channel input corresponds to the source sequences of the previous block, which cannot improve the decoding of the source sequences of the current block at the relay, in contrast to Thm. $8 \mathrm{We}$ conclude that in Thm. 7 we cannot benefit from generating the relay channel input statistically dependent on the common information.

Remark 20. In both Thm.7 and Thm. 8 we used a combination of SW coding and CPM. Since CPM can generally support higher source-channel rates, a natural question that arises is whether it is possible to design a scheme based only on CPM, namely to encode both the cooperation information forwarded by the relay (together with the sources), and the new information transmitted from the sources, using a superposition CPM scheme. This approach cannot be implemented in the framework of the current paper. This follows as the current work uses decoding based on joint typicality, but joint typicality does not apply to different blocks of the same RV. For example, we cannot test the joint typicality of $\mathbf{s}_{b}$ and $\mathbf{s}_{b+1}$, as they belong to different time blocks. Using a CPM-only scheme would require us to carry out such tests. For example, consider the case in which the source pair $\left(S_{1}, S_{2}\right)$ has no common part, that is $\mathcal{T}=\phi$, and also let $\mathcal{Q}=\phi$. Using the CPM technique for sending information to both the relay and the destination would lead to the following relay decoding rule: assume that the relay knows $\left(\mathbf{s}_{1, b-1}, \mathbf{s}_{2, b-1}\right)$ at the end of block $b-1$. The relay decodes $\left(\mathbf{s}_{1, b}, \mathbf{s}_{2, b}\right)$, by looking for a unique pair $\left(\tilde{\mathbf{s}}_{1}, \tilde{\mathbf{s}}_{2}\right) \in \mathcal{S}_{1}^{n} \times \mathcal{S}_{2}^{n}$ such that:

$$
\begin{aligned}
& \left(\tilde{\mathbf{s}}_{1}, \tilde{\mathbf{s}}_{2}, \mathbf{x}_{1}\left(\tilde{\mathbf{s}}_{1}, \mathbf{s}_{1, b-1}\right), \mathbf{x}_{2}\left(\tilde{\mathbf{s}}_{2}, \mathbf{s}_{2, b-1}\right), \mathbf{s}_{1, b-1},\right. \\
& \left.\quad \mathbf{s}_{2, b-1}, \mathbf{x}_{3}\left(\mathbf{s}_{1, b-1}, \mathbf{s}_{2, b-1}\right), \mathbf{w}_{3, b}, \mathbf{y}_{3, b}\right) \in A_{\epsilon}^{*(n)} .
\end{aligned}
$$

Note that $\left(\tilde{\mathbf{s}}_{1}, \tilde{\mathbf{s}}_{2}\right)$ and $\left(\mathbf{s}_{1, b-1}, \mathbf{s}_{2, b-1}\right)$ can not be jointly typical since they correspond to different block indices: $\left(\tilde{\mathbf{s}}_{1}, \tilde{\mathbf{s}}_{2}\right)$ corresponds to block $b$, while $\left(\mathbf{s}_{1, b-1}, \mathbf{s}_{2, b-1}\right)$ corresponds to block $b-1$, and hence, they are independent of each other. Similarly, the destination would require to check typicality across different blocks.

We conclude that a CPM-only scheme cannot be used together with a joint typicality decoder. It may be possible to construct schemes based on a different decoder, or to implement CPM through intermediate RVs to overcome this difficulty, but these topics are left for future research.

Remark 21. A comparison of the decoding rules of Thm. 7 (see Appendix B-C) and Thm. 8 (see Appendix C-C) reveals a difference in the side information block indices used to assist in decoding at the relay and the destination. The decoding rules of Thm. 7 use side information block with the same index as that of the received vector, while the decoding rules of Thm. 8 use side information block with an index earlier than that of the received vector. The difference stems from the fact that in Thm. 7 cooperation between the relay and the sources is achieved via auxiliary RVs which represent bin indices, while in Thm. 8 the cooperation is based on the source sequences. In the DF scheme cooperation information is used with a delay of one block. Therefore, when cooperation is based on the source sequences (Thm. 8), then the side information from the previous block is used for decoding since this is the side information that is correlated with the source sequences.

\section{CONCLUSIONS}

In this paper we considered transmission of arbitrarily correlated sources over MARCs and MABRCs with correlated side information at both the relay and the destination. We first derived an achievable source-channel rate for MARCs based on operational separation, which applies directly to MABRCs as well. This result is established by using an irregular encoding scheme for the channel code. We also showed that for both MABRCs and MARCs regular encoding is more restrictive than irregular encoding. additionally, we obtained necessary conditions for the achievability of sourcechannel rates.

Then, we considered phase fading and Rayleigh fading MARCs with side information and identified conditions under which informational separation is optimal for these channels. Conditions for the optimality of informational separation for fading MABRCs were also obtained. The importance of this result lies in the fact that it supports a modular system design (separate design of the source and channel codes) while achieving the optimal end-to-end performance. We note here that this is the first time that optimality of separation is shown for a MARC or a MABRC configuration.

Lastly, we considered joint source-channel coding for DM MARCs and MABRCs for source-channel rate $\kappa=1$. We presented two new joint source-channel coding schemes for which use a combination of SW source coding and joint sourcechannel coding based on CPM. While in the first scheme CPM is used for encoding information to the relay and SW coding is used for encoding information to the destination; in the second scheme SW coding is used for encoding information to the relay and CPM is used for encoding information to the destination. The different combinations of SW coding and CPM enable flexibility in the system design by choosing one of the two schemes according to the qualities of the side information sequences and received signals at the relay and the destination. In particular, the first scheme generally has looser decoding constraints at the relay, and therefore it is better when the source-relay link is the bottleneck, while the second scheme generally has looser decoding constraints at the destination, and is more suitable to scenarios where the source-destination link is more limiting.

\section{APPENDIX A \\ PROOF of THEOREM 1}

Fix a distribution $p\left(v_{1}\right) p\left(x_{1} \mid v_{1}\right) p\left(v_{2}\right) p\left(x_{2} \mid v_{2}\right) p\left(x_{3} \mid v_{1}, v_{2}\right)$. 


\section{A. Codebook construction}

For $i=1,2$, assign every $\mathbf{s}_{i} \in \mathcal{S}_{i}^{m}$ to one of $2^{m R_{i}^{r}}$ bins independently according to a uniform distribution on $\mathcal{U}_{i}^{r} \triangleq$ $\left\{1,2, \ldots, 2^{m R_{i}^{r}}\right\}$. We refer to these two sets as the relay bins. Denote these assignments by $f_{i}^{r}$. Independent from the relay bin assignments, for $i=1,2$, assign every $\mathbf{s}_{i} \in \mathcal{S}_{i}^{m}$ to one of $2^{m R_{i}^{d}}$ bins independently according to a uniform distribution on $\mathcal{U}_{i}^{d} \triangleq\left\{1,2, \ldots, 2^{m R_{i}^{d}}\right\}$. We refer to these two sets as the destination bins. Denote these assignments by $f_{i}^{d}$.

Next, generate a superposition channel codebook with blocklength $n$, rates $\hat{R}_{i}^{d}=\frac{1}{\kappa} R_{i}^{d}, i=1,2$, auxiliary vectors $\mathbf{v}_{i}\left(u_{i}^{d}\right), u_{i}^{d} \in \mathcal{U}_{i}^{d}, i=1,2$, and channel codewords $\mathbf{x}_{i}\left(u_{i}^{r}, u_{i}^{d}\right)$, $\left(u_{i}^{r}, u_{i}^{d}\right) \in \mathcal{U}_{i}^{r} \times \mathcal{U}_{i}^{d}, i=1,2$, as detailed in [2, Appendix A].

\section{B. Encoding}

Consider the sequences and side information $s_{i, 1}^{B m} \in$ $\mathcal{S}_{i}^{B m}, i=1,2, w_{3,1}^{B m} \in \mathcal{W}_{3}^{B m}$, and $w^{B m} \in \mathcal{W}^{B m}$, all of length $B m$. Partition each sequence into $B$ length $m$ subsequences, $\mathbf{s}_{i, b}, i=1,2, \mathbf{w}_{3, b}$, and $\mathbf{w}_{b}, b=1,2, \ldots, B$. A total of $B m$ source samples are transmitted in $B+1$ blocks of $n$ channel symbols each. For any fixed $(m, n)$ with $n \leq \kappa m$, we can achieve a rate arbitrarily close to $\kappa=n / m$ by increasing $B$, i.e, $(B+1) n / B m \rightarrow \kappa$ as $B \rightarrow \infty$.

At block 1 , transmitter $i, i=1,2$, observes source subsequence $\mathbf{s}_{i, 1}$ and finds its corresponding relay bin index $u_{i, 1}^{r}=$ $f_{i}^{r}\left(\mathbf{s}_{i, 1}\right) \in \mathcal{U}_{u}^{r}$. It transmits the channel codeword $\mathbf{x}_{i}\left(u_{i, 1}^{r}, 1\right)$. In block $b, b=2, \ldots, B$, source terminal $i$ transmits the channel codeword $\mathbf{x}_{i}\left(u_{i, b}^{r}, u_{i, b-1}^{d}\right)$, where $u_{i, b}^{r}=f_{i}^{r}\left(\mathbf{s}_{i, b}\right) \in \mathcal{U}_{i}^{r}$, and $u_{i, b-1}^{d}=f_{i}^{d}\left(\mathbf{s}_{i, b-1}\right) \in \mathcal{U}_{i}^{d}$. In block $B+1$, the source terminal transmits $\mathbf{x}_{i}\left(1, u_{i, B}^{d}\right)$.

At block $b=1$, the relay simply transmits $\mathbf{x}_{3}(1,1)$. Assume that at block $b, b=2, \ldots, B, B+1$, the relay estimates $\left(\mathbf{s}_{1, b-1}, \mathbf{s}_{2, b-1}\right)$. Let $\left(\tilde{\mathbf{s}}_{1, b-1}, \tilde{\mathbf{s}}_{2, b-1}\right)$ denote the estimates. The relay then finds the corresponding destination bin indices $\tilde{u}_{i, b-1}^{d} \in \mathcal{U}_{i}^{d}, i=1,2$, and transmits the channel codeword $\mathbf{x}_{3}\left(\tilde{u}_{1, b-1}^{d}, \tilde{u}_{2, b-1}^{d}\right)$.

\section{Decoding}

The relay decodes the source sequences sequentially trying to reconstruct source blocks $\mathbf{s}_{i, b}, i=1,2$, at the end of channel block $b$ as follows: Let $\left(\tilde{\mathbf{s}}_{1, b-1}, \tilde{\mathbf{s}}_{2, b-1}\right)$ be the estimates of $\left(\mathbf{s}_{1, b-1}, \mathbf{s}_{2, b-1}\right)$ obtained at the end of block $b-1$. Applying $f_{1}^{d}$ and $f_{2}^{d}$, the relay finds the corresponding destination bin indices $\left(\tilde{u}_{1, b-1}^{d}, \tilde{u}_{2, b-1}^{d}\right)$. At time $b$ the relay channel decoder decodes $\left(u_{1, b}^{r}, u_{2, b}^{r}\right)$ by looking for a unique pair $\left(\tilde{u}_{1}^{r}, \tilde{u}_{2}^{r}\right) \in$ $\mathcal{U}_{1}^{r} \times \mathcal{U}_{2}^{r}$ such that:

$\left(\mathbf{v}_{1}\left(\tilde{u}_{1, b-1}^{d}\right), \mathbf{v}_{2}\left(\tilde{u}_{2, b-1}^{d}\right), \mathbf{x}_{1}\left(\tilde{u}_{1}^{r}, \tilde{u}_{1, b-1}^{d}\right), \mathbf{x}_{2}\left(\tilde{u}_{2}^{r}, \tilde{u}_{2, b-1}^{d}\right)\right.$,

$\left.\mathbf{x}_{3}\left(\tilde{u}_{1, b-1}^{d}, \tilde{u}_{2, b-1}^{d}\right), \mathbf{y}_{3, b}\right) \in A_{\epsilon}^{*(n)}\left(V_{1}, V_{2}, X_{1}, X_{2}, X_{3}, Y_{3}\right)$.

The decoded relay bin indices, denoted $\left(\tilde{u}_{1, b}^{r}, \tilde{u}_{2, b}^{r}\right)$, are then given to the relay source decoder, which estimates $\left(\mathbf{s}_{1, b}, \mathbf{s}_{2, b}\right)$. The relay source decoder declares $\left(\tilde{\mathbf{s}}_{1}, \tilde{\mathbf{s}}_{2}\right) \in \mathcal{S}_{1}^{m} \times \mathcal{S}_{2}^{m}$ as the decoded sequences if it is the unique pair of sequences that satisfies $f_{1}^{r}\left(\tilde{\mathbf{s}}_{1}\right)=\tilde{u}_{1, b}^{r}, f_{2}^{r}\left(\tilde{\mathbf{s}}_{2}\right)=\tilde{u}_{2, b}^{r}$ and $\left(\tilde{\mathbf{s}}_{1}, \tilde{\mathbf{s}}_{2}, \mathbf{w}_{3, b}\right) \in$ $A_{\epsilon}^{*(m)}\left(S_{1}, S_{2}, W_{3}\right)$. The decoded sequences are denoted by $\left(\tilde{\mathbf{s}}_{1, b}, \tilde{\mathbf{s}}_{2, b}\right)$.
Decoding at the destination is done using backward decoding. The destination node waits until the end of channel block $B+1$. It first tries to decode $\left(\mathbf{s}_{1, B}, \mathbf{s}_{2, B}\right)$ using the received signal at channel block $B+1$ and its side information $\mathbf{w}_{B}$. Going backwards from the last channel block to the first, we assume that the destination has estimates $\left(\hat{\mathbf{s}}_{1, b+1}, \hat{\mathbf{s}}_{2, b+1}\right)$ of $\left(\mathbf{s}_{1, b+1}, \mathbf{s}_{2, b+1}\right)$, and consider decoding of $\left(\mathbf{s}_{1, b}, \mathbf{s}_{2, b}\right)$. From $\hat{\mathbf{s}}_{i, b+1}, i=1,2$, the destination finds the relay bin indices $\hat{u}_{i, b+1}^{r}=f_{i}^{r}\left(\hat{\mathbf{s}}_{i, b+1}\right)$. At block $b+1$ the destination channel decoder first estimates the destination bin indices $\left(u_{1, b}^{d}, u_{2, b}^{d}\right)$ by looking for a unique pair $\left(\hat{u}_{1}^{d}, \hat{u}_{2}^{d}\right) \in \mathcal{U}_{1}^{d} \times \mathcal{U}_{2}^{d}$ such that:

$$
\begin{aligned}
& \left(\mathbf{v}_{1}\left(\hat{u}_{1}^{d}\right), \mathbf{v}_{2}\left(\hat{u}_{2}^{d}\right), \mathbf{x}_{1}\left(\hat{u}_{1, b+1}^{r}, \hat{u}_{1}^{d}\right), \mathbf{x}_{2}\left(\hat{u}_{2, b+1}^{r}, \hat{u}_{2}^{d}\right),\right. \\
& \left.\quad \mathbf{x}_{3}\left(\hat{u}_{1}^{d}, \hat{u}_{2}^{d}\right), \mathbf{y}_{b+1}\right) \in A_{\epsilon}^{*(n)}\left(V_{1}, V_{2}, X_{1}, X_{2}, X_{3}, Y_{3}\right) .
\end{aligned}
$$

The decoded destination bin indices, denoted $\left(\hat{u}_{1, b}^{d}, \hat{u}_{2, b}^{d}\right)$, are then given to the destination source decoder, which estimates the source sequences $\left(\mathbf{s}_{1, b}, \mathbf{s}_{2, b}\right)$. The destination source decoder declares $\left(\hat{\mathbf{s}}_{1}, \hat{\mathbf{s}}_{2}\right) \in \mathcal{S}_{1}^{m} \times \mathcal{S}_{2}^{m}$ as the decoded sequences if it is the unique pair of sequences that satisfies $f_{1}^{d}\left(\hat{\mathbf{s}}_{1}\right)=$ $\hat{u}_{1, b}^{d}, f_{2}^{d}\left(\hat{\mathbf{s}}_{2}\right)=\hat{u}_{2, b}^{d}$ and $\left(\hat{\mathbf{s}}_{1}, \hat{\mathbf{s}}_{2}, \mathbf{w}_{b}\right) \in A_{\epsilon}^{*(m)}\left(S_{1}, S_{2}, W\right)$. The decoded sequences are denoted by $\left(\hat{\mathbf{s}}_{1, b}, \hat{\mathbf{s}}_{2, b}\right)$.

\section{Error Probability Analysis}

Using standard techniques it can be shown that decoding the source sequences at the relay can be done reliably as long as $8 \mathrm{a}-18 \mathrm{c}$ ) hold, and decoding the source sequences

\section{APPENDIX B \\ PROOF OF THEOREM 7}

Fix a distribution $p\left(s_{1}, s_{2}, w_{3}, w\right) p(q) p\left(v_{1}\right) p\left(x_{1} \mid s_{1}, v_{1}, q\right)$ $p\left(v_{2}\right) p\left(x_{2} \mid s_{2}, v_{2}, q\right) p\left(x_{3} \mid v_{1}, v_{2}\right) p\left(y_{3}, y \mid x_{1}, x_{2}, x_{3}\right)$.

\section{A. Codebook construction}

For $i=1,2$, assign every $\mathbf{s}_{i} \in \mathcal{S}_{i}^{n}$ to one of $2^{n R_{i}}$ bins independently according to a uniform distribution on $\mathcal{U}_{i} \triangleq$ $\left\{1,2, \ldots, 2^{n R_{i}}\right\}$. Denote this assignment by $f_{i}, i=1,2$.

For the channel codebook, for each $i=1,2$, generate $2^{n R_{i}}$ codewords $\mathbf{v}_{i}\left(u_{i}\right), u_{i} \in \mathcal{U}_{i}$, by choosing the letters $v_{i, k}\left(u_{i}\right), k=1,2, \ldots, n$, independently according to the distribution $p_{V_{i}}\left(v_{i, k}\left(u_{i}\right)\right)$. For each $\mathbf{t} \in \mathcal{T}^{n}$ generate one length $n$ codeword $\mathbf{q}(\mathbf{t})$ by choosing the letters $q_{k}$ independently with distribution $p_{Q}\left(q_{k}\right), k=1,2, \ldots, n$. For each pair $\left(\mathbf{s}_{i}, u_{i}\right) \in \mathcal{S}_{i}^{n} \times \mathcal{U}_{i}, i=1,2$, find the corresponding $\mathbf{t}=$ $h_{i}\left(\mathbf{s}_{i}\right)$, and generate one length $n$ codeword $\mathbf{x}_{i}\left(\mathbf{s}_{i}, u_{i}, \mathbf{q}(\mathbf{t})\right)$, $\mathbf{q} \in \mathcal{Q}^{n}$, by choosing the letters $x_{i, k}\left(\mathbf{s}_{i}, u_{i}, \mathbf{q}(\mathbf{t})\right)$ independently with distribution $p_{X_{i} \mid S_{i}, V_{i}, Q}\left(x_{i, k} \mid s_{i, k}, v_{i, k}\left(u_{i}\right), q_{k}(\mathbf{t})\right)$, $k=1,2, \ldots, n$. Finally, generate one length- $n$ relay codeword $\mathbf{x}_{3}\left(u_{1}, u_{2}\right)$ for each pair $\left(u_{1}, u_{2}\right) \in \mathcal{U}_{1} \times \mathcal{U}_{2}$ by choosing $x_{3, k}\left(u_{1}, u_{2}\right)$ independently with distribution $p_{X_{3} \mid V_{1}, V_{2}}\left(x_{3, k} \mid v_{1, k}\left(u_{1}\right), v_{2, k}\left(u_{2}\right)\right), k=1,2, \ldots, n$.

\section{B. Encoding}

Consider the sequences $s_{i, 1}^{B n} \in \mathcal{S}_{i}^{B n}, i=1,2, w_{3,1}^{B n} \in \mathcal{W}_{3}^{B n}$, and $w^{B n} \in \mathcal{W}^{B n}$, all of length $B n$. Partition each sequence into $B$ length- $n$ subsequences, $\mathbf{s}_{i, b}, i=1,2, \mathbf{w}_{3, b}$, and 
$\mathbf{w}_{b}, b=1,2, \ldots, B$. A total of $B n$ source samples are transmitted in $B+1$ blocks of $n$ channel symbols each. At block 1 , source terminal $i, i=1,2$, finds $\mathbf{t}_{i}=h_{i}\left(\mathbf{s}_{i, 1}\right)$, and transmits the channel codeword $\mathbf{x}_{i}\left(\mathbf{s}_{i, 1}, 1, \mathbf{q}\left(h_{i}\left(\mathbf{s}_{i, 1}\right)\right)\right)$. At block $b, b=2, \ldots, B$, source terminal $i, i=1,2$, transmits the channel codeword $\mathbf{x}_{i}\left(\mathbf{s}_{i, b}, u_{i, b-1}, \mathbf{q}\left(h_{i}\left(\mathbf{s}_{i, b}\right)\right)\right)$, where $u_{i, b-1}=f_{i}\left(\mathbf{s}_{i, b-1}\right) \in \mathcal{U}_{i}$ is the bin index of source vector $\mathbf{s}_{i, b-1}$. Let $\left(\mathbf{a}_{1}, \mathbf{a}_{2}\right) \in \mathcal{S}_{1}^{n} \times \mathcal{S}_{2}^{n}$ be two sequences generated i.i.d according to $p\left(\mathbf{a}_{1}, \mathbf{a}_{2}\right)=\prod_{k=1}^{n} p_{S_{1}, S_{2}}\left(a_{1, k}, a_{2, k}\right)$. These sequences are known to all nodes. At block $B+1$, source terminal $i$ transmits $\mathbf{x}_{i}\left(\mathbf{a}_{i}, u_{i, B}, \mathbf{q}\left(h_{i}\left(\mathbf{a}_{i}\right)\right)\right)$.

At block $b=1$, the relay transmits $\mathbf{x}_{3}(1,1)$. Assume that at block $b, b=2, \ldots, B, B+1$, the relay has estimates $\left(\tilde{\mathbf{s}}_{1, b-1}, \tilde{\mathbf{s}}_{2, b-1}\right)$ of $\left(\mathbf{s}_{1, b-1}, \mathbf{s}_{2, b-1}\right)$. It then finds the corresponding bin indices $\tilde{u}_{i, b-1}=f_{i}\left(\tilde{\mathbf{s}}_{1, b-1}\right) \in \mathcal{U}_{i}, i=1,2$, and transmits the channel codeword $\mathbf{x}_{3}\left(\tilde{u}_{1, b-1}, \tilde{u}_{2, b-1}\right)$.

\section{Decoding}

The relay decodes the source sequences sequentially, trying to reconstruct $\left(\mathbf{s}_{1, b}, \mathbf{s}_{2, b}\right)$ at the end of channel block $b$ as follows: Let $\left(\tilde{\mathbf{s}}_{1, b-1}, \tilde{\mathbf{s}}_{2, b-1}\right)$ be the estimates of $\left(\mathbf{s}_{1, b-1}, \mathbf{s}_{2, b-1}\right)$ obtained at the end of block $b-1$. The relay thus knows the corresponding bin indices $\left(\tilde{u}_{1, b-1}, \tilde{u}_{2, b-1}\right)$. Using this information, its received signal $\mathbf{y}_{3, b}$, and the side information $\mathbf{w}_{3, b}$, the relay decodes $\left(\mathbf{s}_{1, b}, \mathbf{s}_{2, b}\right)$, by looking for a unique pair $\left(\tilde{\mathbf{s}}_{1}, \tilde{\mathbf{s}}_{2}\right) \in \mathcal{S}_{1}^{n} \times \mathcal{S}_{2}^{n}$ such that:

$$
\begin{aligned}
& \left(\tilde{\mathbf{s}}_{1}, \tilde{\mathbf{s}}_{2}, \tilde{\mathbf{t}}, \mathbf{q}(\tilde{\mathbf{t}}), \mathbf{v}_{1}\left(\tilde{u}_{1, b-1}\right), \mathbf{v}_{2}\left(\tilde{u}_{2, b-1}\right), \mathbf{x}_{1}\left(\tilde{\mathbf{s}}_{1}, \tilde{u}_{1, b-1}, \mathbf{q}(\tilde{\mathbf{t}})\right),\right. \\
& \left.\quad \mathbf{x}_{2}\left(\tilde{\mathbf{s}}_{2}, \tilde{u}_{2, b-1}, \mathbf{q}(\tilde{\mathbf{t}})\right), \mathbf{x}_{3}\left(\tilde{u}_{1, b-1}, \tilde{u}_{2, b-1}\right), \mathbf{w}_{3, b}, \mathbf{y}_{3, b}\right) \\
& \left.\quad \in A_{\epsilon}^{*(n)}\left(S_{1}, S_{2}, T, Q, V_{1}, V_{2}, X_{1}, X_{2}, X_{3}, W_{3}, Y_{3}\right), \quad \text { B. } 1\right)
\end{aligned}
$$

where $\tilde{\mathbf{t}}=h_{1}\left(\tilde{\mathbf{s}}_{1}\right)=h_{2}\left(\tilde{\mathbf{s}}_{2}\right)$. Denote the decoded sequence $\left(\tilde{\mathbf{s}}_{1, b}, \tilde{\mathbf{s}}_{2, b}\right)$.

Decoding at the destination is done using backward decoding. Let $\boldsymbol{\alpha} \in \mathcal{W}^{n}$ be a sequence generated i.i.d according to $p_{W \mid S_{1}, S_{2}}\left(\alpha_{k} \mid a_{1, k}, a_{2, k}\right), k=1,2, \ldots, n$. The destination node waits until the end of channel block $B+1$. It first tries to decode $\left(\mathbf{s}_{1, B}, \mathbf{s}_{2, B}\right)$ using the received signal at channel block $B+1$ and $\boldsymbol{\alpha}$. Going backwards from the last channel block to the first, we assume that the destination has estimates $\left(\hat{\mathbf{s}}_{1, b+1}, \hat{\mathbf{s}}_{2, b+1}\right)$ of $\left(\mathbf{s}_{1, b+1}, \mathbf{s}_{2, b+1}\right)$, and therefore has the estimates $\hat{\mathbf{t}}_{b+1}=h_{1}\left(\hat{\mathbf{s}}_{1, b+1}\right)=h_{2}\left(\hat{\mathbf{s}}_{2, b+1}\right)$ and $\mathbf{q}\left(\hat{\mathbf{t}}_{b+1}\right)$. At block $b+1$ the destination channel decoder first estimates the destination bin indices $\hat{u}_{i, b}, i=1,2$, corresponding to $\mathbf{s}_{i, b}$, based on its received signal $\mathbf{y}_{b+1}$ and the side information $\mathbf{w}_{b+1}$, by looking for a unique pair $\left(\hat{u}_{1}, \hat{u}_{2}\right) \in \mathcal{U}_{1} \times \mathcal{U}_{2}$ such that:

$$
\begin{aligned}
& \left(\hat{\mathbf{s}}_{1, b+1}, \hat{\mathbf{s}}_{2, b+1}, \hat{\mathbf{t}}_{b+1}, \mathbf{q}\left(\hat{\mathbf{t}}_{b+1}\right), \mathbf{v}_{1}\left(\hat{u}_{1}\right), \mathbf{v}_{2}\left(\hat{u}_{2}\right),\right. \\
& \quad \mathbf{x}_{1}\left(\hat{\mathbf{s}}_{1, b+1}, \hat{u}_{1}, \mathbf{q}\left(\hat{\mathbf{t}}_{b+1}\right)\right), \mathbf{x}_{2}\left(\hat{\mathbf{s}}_{2, b+1}, \hat{u}_{2}, \mathbf{q}\left(\hat{\mathbf{t}}_{b+1}\right)\right), \\
& \left.\quad \mathbf{x}_{3}\left(\hat{u}_{1}, \hat{u}_{2}\right), \mathbf{w}_{b+1}, \mathbf{y}_{b+1}\right) \\
& \quad \in A_{\epsilon}^{*(n)}\left(S_{1}, S_{2}, T, Q, V_{1}, V_{2}, X_{1}, X_{2}, X_{3}, W, Y\right) .
\end{aligned}
$$

The decoded destination bin indices, denoted by $\left(\hat{u}_{1, b}, \hat{u}_{2, b}\right)$, are then given to the destination source decoder, which estimates $\left(\mathbf{s}_{1, b}, \mathbf{s}_{2, b}\right)$ by looking for a unique pair of sequences $\left(\hat{\mathbf{s}}_{1}, \hat{\mathbf{s}}_{2}\right) \in \mathcal{S}_{1}^{n} \times \mathcal{S}_{2}^{n}$ that satisfies $f_{1}\left(\hat{\mathbf{s}}_{1}\right)=\hat{u}_{1, b}, f_{2}\left(\hat{\mathbf{s}}_{2}\right)=\hat{u}_{2, b}$ and $\left(\hat{\mathbf{s}}_{1}, \hat{\mathbf{s}}_{2}, \mathbf{w}_{b}\right) \in A_{\epsilon}^{*(n)}\left(S_{1}, S_{2}, W\right)$. The decoded sequences are denoted by $\left(\hat{\mathbf{s}}_{1, b}, \hat{\mathbf{s}}_{1, b}\right)$.

\section{Error Probability Analysis}

We start with the relay error probability analysis. Let $E_{b}^{r}\left(\mathbf{s}_{1, b}, \mathbf{s}_{2, b} ; u_{1, b-1}, u_{2, b-1}\right)$ denote the relay decoding error event in block $b$, assuming $\left(u_{1, b-1}, u_{2, b-1}\right)$ are available at the relay, and $\left(\mathbf{s}_{1, b}, \mathbf{s}_{2, b}\right)$ are the source sequences at block $b$. Thus, this error event is the event that $\left(\tilde{\mathbf{s}}_{1, b}, \tilde{\mathbf{s}}_{2, b}\right) \neq$ $\left(\mathbf{s}_{1, b}, \mathbf{s}_{2, b}\right)$. Let $\mathscr{D}_{b}$ denote the event that $\left(\mathbf{s}_{1, b}, \mathbf{s}_{2, b}, \mathbf{w}_{3, b}, \mathbf{t}_{b}\right) \in$ $A_{\epsilon}^{*(n)}\left(S_{1}, S_{2}, W_{3}, T\right)$. The average decoding error probability at the relay in block $b, \bar{P}_{r}^{(n)}$, is defined in $\mathrm{B} .3$ at the bottom of the page. In the following we show that the inner sum in (B.3) can be upper bounded independently of $\left(u_{1, b-1}, u_{2, b-1}\right)$. Therefore, for any fixed value of $\left(u_{1, b-1}, u_{2, b-1}\right)$ we have (B.4) at the bottom of the page, where B.4a follows from the union bound and $\mathrm{B} .4 \mathrm{~b}$ follows from the AEP [37, Ch. 5.1], for sufficiently large $n$, and as $\mathbf{t}_{b}$ is a deterministic function of $\left(\mathbf{s}_{1, b}, \mathbf{s}_{2, b}\right)$. This deterministic relationship implies that $\left(\mathbf{s}_{1, b}, \mathbf{s}_{2, b}, \mathbf{w}_{3, b}\right) \in A_{\epsilon}^{*(n)}\left(S_{1}, S_{2}, W_{3}\right)$ if and only if $\left(\mathbf{s}_{1, b}, \mathbf{s}_{2, b}, \mathbf{w}_{3, b}, \mathbf{t}_{b}\right) \in A_{\epsilon}^{*(n)}\left(S_{1}, S_{2}, W_{3}, T\right)$. Note also that

$$
\bar{P}_{r}^{(n)} \triangleq \sum_{\left(u_{1, b-1}, u_{2, b-1}\right) \in \mathcal{U}_{1} \times \mathcal{U}_{2}} p\left(u_{1, b-1}, u_{2, b-1}\right) \sum_{\left(\mathbf{s}_{1, b}, \mathbf{s}_{2, b}\right) \in \mathcal{S}_{1}^{n} \times \mathcal{S}_{2}^{n}} p\left(\mathbf{s}_{1, b}, \mathbf{s}_{2, b}\right) \operatorname{Pr}\left\{E_{b}^{r}\left(\mathbf{s}_{1, b}, \mathbf{s}_{2, b} ; u_{1, b-1}, u_{2, b-1}\right)\right\} .
$$

$$
\begin{aligned}
& \sum_{\left(\mathbf{s}_{1, b}, \mathbf{s}_{2, b}\right) \in \mathcal{S}_{1}^{n} \times \mathcal{S}_{2}^{n}} p\left(\mathbf{s}_{1, b}, \mathbf{s}_{2, b}\right) \operatorname{Pr}\left\{E_{b}^{r}\left(\mathbf{s}_{1, b}, \mathbf{s}_{2, b} ; u_{1, b-1}, u_{2, b-1}\right)\right\} \\
& \quad \leq \sum_{\left(\mathbf{s}_{1, b}, \mathbf{s}_{2, b}, \mathbf{w}_{3, b}\right) \notin A_{\epsilon}^{*(n)}\left(S_{1}, S_{2}, W_{3}\right)} p\left(\mathbf{s}_{1, b}, \mathbf{s}_{2, b}, \mathbf{w}_{3, b}\right) \\
& \quad+\sum_{\substack{\left(\mathbf{s}_{1, b}, \mathbf{s}_{2, b}, \mathbf{w}_{3, b}\right) \in A_{\epsilon}^{*(n)} \\
\leq}} p\left(\mathbf{s}_{1, b}, \mathbf{s}_{2, b}, \mathbf{w}_{3, b}\right) \operatorname{Pr}\left\{E_{b}^{r}\left(\mathbf{s}_{1, b}, \mathbf{s}_{2, b} ; u_{1, b-1}, u_{2, b-1}\right) \mid\left(\mathbf{s}_{1, b}, \mathbf{s}_{2, b}, \mathbf{w}_{3, b}\right) \in A_{\epsilon}^{*(n)}\left(S_{1}, S_{2}, W_{3}\right)\right\} \\
& \quad \sum_{\left(\mathbf{s}_{1, b}, \mathbf{s}_{2, b}, \mathbf{w}_{3, b}, \mathbf{t}_{b}\right) \in A_{\epsilon}^{*(n)}\left(S_{1}, S_{2}, W_{3}, T\right)} p\left(\mathbf{s}_{1, b}, \mathbf{s}_{2, b}, \mathbf{w}_{3, b}\right) \operatorname{Pr}\left\{E_{b}^{r}\left(\mathbf{s}_{1, b}, \mathbf{s}_{2, b} ; u_{1, b-1}, u_{2, b-1}\right) \mid \mathscr{D}_{b}\right\}
\end{aligned}
$$


(B.4b) follows similarly to [7, Eq. (16)]. Next, we show that for $\left(\mathbf{s}_{1, b}, \mathbf{s}_{2, b}, \mathbf{w}_{3, b}, \mathbf{t}_{b}\right) \in A_{\epsilon}^{*(n)}\left(S_{1}, S_{2}, W_{3}, T\right)$, the summands in B.4b can be upper bounded independently of $\left(\mathbf{s}_{1, b}, \mathbf{s}_{2, b}, \mathbf{w}_{3, b}\right)$, for any fixed value of $\left(u_{1, b-1}, u_{2, b-1}\right)$.

Let $\epsilon_{0}, \epsilon_{1}$ and $\epsilon_{2}$ be positive numbers such that $\epsilon_{0} \geq \epsilon_{2} \geq$ $\epsilon_{1}>\epsilon$ and $\epsilon_{0} \rightarrow 0$ as $\epsilon \rightarrow 0$. Assuming correct decoding at block $b-1$ (hence $\left(u_{1, b-1}, u_{2, b-1}\right)$ are available at the relay), we define the following events:

$$
\begin{gathered}
E_{1}^{r} \triangleq\left\{\left(\mathbf{s}_{1, b}, \mathbf{s}_{2, b}, \mathbf{t}_{b}, \mathbf{Q}\left(\mathbf{t}_{b}\right), \mathbf{V}_{1}\left(u_{1, b-1}\right), \mathbf{V}_{2}\left(u_{2, b-1}\right),\right.\right. \\
\mathbf{X}_{1}\left(\mathbf{s}_{1, b}, u_{1, b-1}, \mathbf{Q}\left(\mathbf{t}_{b}\right)\right), \mathbf{X}_{2}\left(\mathbf{s}_{2, b}, u_{2, b-1}, \mathbf{Q}\left(\mathbf{t}_{b}\right)\right), \\
\left.\left.\mathbf{X}_{3}\left(u_{1, b-1}, u_{2, b-1}\right), \mathbf{w}_{3, b}, \mathbf{Y}_{3, b}\right) \notin A_{\epsilon}^{*(n)}\right\}, \\
E_{2}^{r} \triangleq\left\{\exists\left(\tilde{\mathbf{s}}_{1}, \tilde{\mathbf{s}}_{2}\right) \in \mathcal{S}_{1}^{n} \times \mathcal{S}_{2}^{n}:\right. \\
\left(\tilde{\mathbf{s}}_{1}, \tilde{\mathbf{s}}_{2}\right) \neq\left(\mathbf{s}_{1, b}, \mathbf{s}_{2, b}\right), \tilde{\mathbf{t}}=h_{1}\left(\tilde{\mathbf{s}}_{1}\right)=h_{2}\left(\tilde{\mathbf{s}}_{2}\right), \\
\left(\tilde{\mathbf{s}}_{1}, \tilde{\mathbf{s}}_{2}, \tilde{\mathbf{t}}, \mathbf{Q}(\tilde{\mathbf{t}}), \mathbf{V}_{1}\left(u_{1, b-1}\right), \mathbf{V}_{2}\left(u_{2, b-1}\right),\right. \\
\mathbf{X}_{1}\left(\tilde{\mathbf{s}}_{1}, u_{1, b-1}, \mathbf{Q}(\tilde{\mathbf{t}})\right), \mathbf{X}_{2}\left(\tilde{\mathbf{s}}_{2}, u_{2, b-1}, \mathbf{Q}(\tilde{\mathbf{t}})\right), \\
\left.\left.\mathbf{X}_{3}\left(u_{1, b-1}, u_{2, b-1}\right), \mathbf{w}_{3, b}, \mathbf{Y}_{3, b}\right) \in A_{\epsilon}^{*(n)}\right\} .
\end{gathered}
$$

From the AEP [37, Ch. 5.1], for sufficiently large $n$, $\operatorname{Pr}\left\{E_{1}^{r} \mid \mathscr{D}_{b}\right\} \leq \epsilon$. Therefore we can bound

$$
\begin{array}{r}
\operatorname{Pr}\left\{E_{b}^{r}\left(\mathbf{s}_{1, b}, \mathbf{s}_{2, b} ; u_{1, b-1}, u_{2, b-1}\right) \mid \mathscr{D}_{b}\right\} \\
\leq \epsilon+\operatorname{Pr}\left\{E_{2}^{r} \mid\left(E_{1}^{r}\right)^{c}\right\} .
\end{array}
$$

The event $E_{2}^{r}$ is the union of the following events:

$$
\begin{aligned}
& E_{21}^{r} \triangleq\left\{\exists \tilde{\mathbf{s}}_{1} \in \mathcal{S}_{1}^{n}: \tilde{\mathbf{s}}_{1} \neq \mathbf{s}_{1, b}, h_{1}\left(\tilde{\mathbf{s}}_{1}\right)=h_{2}\left(\mathbf{s}_{2, b}\right)=\mathbf{t}_{b}\right. \text {, } \\
& \left(\tilde{\mathbf{s}}_{1}, \mathbf{s}_{2, b}, \mathbf{t}_{b}, \mathbf{Q}\left(\mathbf{t}_{b}\right), \mathbf{V}_{1}\left(u_{1, b-1}\right), \mathbf{V}_{2}\left(u_{2, b-1}\right),\right. \\
& \mathbf{X}_{1}\left(\tilde{\mathbf{s}}_{1}, u_{1, b-1}, \mathbf{Q}\left(\mathbf{t}_{b}\right)\right), \mathbf{X}_{2}\left(\mathbf{s}_{2, b}, u_{2, b-1}, \mathbf{Q}\left(\mathbf{t}_{b}\right)\right) \text {, } \\
& \left.\left.\mathbf{X}_{3}\left(u_{1, b-1}, u_{2, b-1}\right), \mathbf{w}_{3, b}, \mathbf{Y}_{3, b}\right) \in A_{\epsilon}^{*(n)}\right\} \\
& E_{22}^{r} \triangleq\left\{\exists \tilde{\mathbf{s}}_{2} \in \mathcal{S}_{2}^{n}: \tilde{\mathbf{s}}_{2} \neq \mathbf{s}_{2, b}, h_{1}\left(\mathbf{s}_{1, b}\right)=h_{2}\left(\tilde{\mathbf{s}}_{2}\right)=\mathbf{t}_{b}\right. \text {, } \\
& \left(\mathbf{s}_{1, b}, \tilde{\mathbf{s}}_{2},, \mathbf{t}_{b}, \mathbf{Q}\left(\mathbf{t}_{b}\right), \mathbf{V}_{1}\left(u_{1, b-1}\right), \mathbf{V}_{2}\left(u_{2, b-1}\right),\right. \\
& \mathbf{X}_{1}\left(\mathbf{s}_{1, b}, u_{1, b-1}, \mathbf{Q}\left(\mathbf{t}_{b}\right)\right), \mathbf{X}_{2}\left(\tilde{\mathbf{s}}_{2}, u_{2, b-1}, \mathbf{Q}\left(\mathbf{t}_{b}\right)\right) \text {, } \\
& \left.\left.\mathbf{X}_{3}\left(u_{1, b-1}, u_{2, b-1}\right), \mathbf{w}_{3, b}, \mathbf{Y}_{3, b}\right) \in A_{\epsilon}^{*(n)}\right\} \\
& E_{23}^{r} \triangleq\left\{\exists\left(\tilde{\mathbf{s}}_{1}, \tilde{\mathbf{s}}_{2}\right) \in \mathcal{S}_{1}^{n} \times \mathcal{S}_{2}^{n}:\right. \\
& \tilde{\mathbf{s}}_{1} \neq \mathbf{s}_{1, b}, \tilde{\mathbf{s}}_{2} \neq \mathbf{s}_{2, b}, h_{1}\left(\tilde{\mathbf{s}}_{1}\right)=h_{2}\left(\tilde{\mathbf{s}}_{2}\right)=\mathbf{t}_{b}, \\
& \left(\tilde{\mathbf{s}}_{1}, \tilde{\mathbf{s}}_{2}, \mathbf{t}_{b}, \mathbf{Q}\left(\mathbf{t}_{b}\right), \mathbf{V}_{1}\left(u_{1, b-1}\right), \mathbf{V}_{2}\left(u_{2, b-1}\right),\right. \\
& \mathbf{X}_{1}\left(\tilde{\mathbf{s}}_{1}, u_{1, b-1}, \mathbf{Q}\left(\mathbf{t}_{b}\right)\right), \mathbf{X}_{2}\left(\tilde{\mathbf{s}}_{2}, u_{2, b-1}, \mathbf{Q}\left(\mathbf{t}_{b}\right)\right) \\
& \left.\left.\mathbf{X}_{3}\left(u_{1, b-1}, u_{2, b-1}\right), \mathbf{w}_{3, b}, \mathbf{Y}_{3, b}\right) \in A_{\epsilon}^{*(n)}\right\} \\
& E_{24}^{r} \triangleq\left\{\exists\left(\tilde{\mathbf{s}}_{1}, \tilde{\mathbf{s}}_{2}\right) \in \mathcal{S}_{1}^{n} \times \mathcal{S}_{2}^{n}: \tilde{\mathbf{s}}_{1} \neq \mathbf{s}_{1, b}, \tilde{\mathbf{s}}_{2} \neq \mathbf{s}_{2, b},\right. \\
& h_{1}\left(\tilde{\mathbf{s}}_{1}\right)=h_{2}\left(\tilde{\mathbf{s}}_{2}\right)=\tilde{\mathbf{t}} \neq \mathbf{t}_{b}, \mathbf{Q}(\tilde{\mathbf{t}}) \neq \mathbf{Q}\left(\mathbf{t}_{b}\right) \text {, } \\
& \left(\tilde{\mathbf{s}}_{1}, \tilde{\mathbf{s}}_{2}, \tilde{\mathbf{t}}, \mathbf{Q}(\tilde{\mathbf{t}}), \mathbf{V}_{1}\left(u_{1, b-1}\right), \mathbf{V}_{2}\left(u_{2, b-1}\right),\right. \\
& \mathbf{X}_{1}\left(\tilde{\mathbf{s}}_{1}, u_{1, b-1}, \mathbf{Q}(\tilde{\mathbf{t}})\right), \mathbf{X}_{2}\left(\tilde{\mathbf{s}}_{2}, u_{2, b-1}, \mathbf{Q}(\tilde{\mathbf{t}})\right), \\
& \left.\left.\mathbf{X}_{3}\left(u_{1, b-1}, u_{2, b-1}\right), \mathbf{w}_{3, b}, \mathbf{Y}_{3, b}\right) \in A_{\epsilon}^{*(n)}\right\} \\
& E_{25}^{r} \triangleq\left\{\exists\left(\tilde{\mathbf{s}}_{1}, \tilde{\mathbf{s}}_{2}\right) \in \mathcal{S}_{1}^{n} \times \mathcal{S}_{2}^{n}: \tilde{\mathbf{s}}_{1} \neq \mathbf{s}_{1, b}, \tilde{\mathbf{s}}_{2} \neq \mathbf{s}_{2, b},\right. \\
& h_{1}\left(\tilde{\mathbf{s}}_{1}\right)=h_{2}\left(\tilde{\mathbf{s}}_{2}\right)=\tilde{\mathbf{t}} \neq \mathbf{t}_{b}, \mathbf{Q}(\tilde{\mathbf{t}})=\mathbf{Q}\left(\mathbf{t}_{b}\right), \\
& \left(\tilde{\mathbf{s}}_{1}, \tilde{\mathbf{s}}_{2}, \tilde{\mathbf{t}}, \mathbf{Q}(\tilde{\mathbf{t}}), \mathbf{V}_{1}\left(u_{1, b-1}\right), \mathbf{V}_{2}\left(u_{2, b-1}\right),\right. \\
& \mathbf{X}_{1}\left(\tilde{\mathbf{s}}_{1}, u_{1, b-1}, \mathbf{Q}(\tilde{\mathbf{t}})\right), \mathbf{X}_{2}\left(\tilde{\mathbf{s}}_{2}, u_{2, b-1}, \mathbf{Q}(\tilde{\mathbf{t}})\right), \\
& \left.\left.\mathbf{X}_{3}\left(u_{1, b-1}, u_{2, b-1}\right), \mathbf{w}_{3, b}, \mathbf{Y}_{3, b}\right) \in A_{\epsilon}^{*(n)}\right\} .
\end{aligned}
$$

Hence, by the union bound it follows that $\operatorname{Pr}\left\{E_{2}^{r} \mid\left(E_{1}^{r}\right)^{c}\right\}=$ $\sum_{j=1}^{5} \operatorname{Pr}\left\{E_{2 j}^{r} \mid\left(E_{1}^{r}\right)^{c}\right\}$. To bound $\operatorname{Pr}\left\{E_{21}^{r} \mid\left(E_{1}^{r}\right)^{c}\right\}$ we first define the event $E_{21}^{r}\left(\tilde{\mathbf{s}}_{1}\right)$ as follows

$$
\begin{aligned}
E_{21}^{r}\left(\tilde{\mathbf{s}}_{1}\right) \triangleq & \left\{h_{1}\left(\tilde{\mathbf{s}}_{1}\right)=h_{2}\left(\mathbf{s}_{2, b}\right)=\mathbf{t}_{b},\right. \\
& \left(\tilde{\mathbf{s}}_{1}, \mathbf{s}_{2, b}, \mathbf{t}_{b}, \mathbf{Q}\left(\mathbf{t}_{b}\right), \mathbf{V}_{1}\left(u_{1, b-1}\right), \mathbf{V}_{2}\left(u_{2, b-1}\right),\right. \\
& \mathbf{X}_{1}\left(\tilde{\mathbf{s}}_{1}, u_{1, b-1}, \mathbf{Q}\left(\mathbf{t}_{b}\right)\right), \mathbf{X}_{2}\left(\mathbf{s}_{2, b}, u_{2, b-1}, \mathbf{Q}\left(\mathbf{t}_{b}\right)\right), \\
& \left.\left.\left.\mathbf{X}_{3}\left(u_{1, b-1}, u_{2, b-1}\right), \mathbf{w}_{3, b}, \mathbf{Y}_{3, b}\right) \in A_{\epsilon}^{*(n)}\right\} . \quad \text { B } 6\right)
\end{aligned}
$$

Recalling that for $E_{21}^{r}$ then $\tilde{\mathbf{s}}_{1} \neq \mathbf{s}_{1, b}$, we have

$$
\operatorname{Pr}\left\{E_{21}^{r} \mid\left(E_{1}^{r}\right)^{c}\right\}=\sum_{\substack{\tilde{\mathbf{s}}_{1} \neq \mathbf{s}_{1, b}, \tilde{\mathbf{s}}_{1} \in A_{\epsilon}^{*(n)}\left(S_{1} \mid \mathbf{s}_{2, b}, \mathbf{w}_{3, b}, \mathbf{t}_{b}\right)}} \operatorname{Pr}\left\{E_{21}^{r}\left(\tilde{\mathbf{s}}_{1}\right) \mid\left(E_{1}^{r}\right)^{c}\right\} .
$$

Note that in B.77 we consider $\left.\tilde{\mathbf{s}}_{1} \in A_{\epsilon}^{*(n)}\left(S_{1} \mid \mathbf{s}_{2, b}, \mathbf{w}_{3, b}, \mathbf{t}_{b}\right)\right\}$, as otherwise $\operatorname{Pr}\left\{E_{21}^{r}\left(\tilde{\mathbf{s}}_{1}\right) \mid\left(E_{1}^{r}\right)^{c}\right\}=0$. Therefore, in the following we upper bound $\operatorname{Pr}\left\{E_{21}^{r}\left(\tilde{\mathbf{s}}_{1}\right) \mid\left(E_{1}^{r}\right)^{c}, \tilde{\mathbf{s}}_{1} \in\right.$ $\left.A_{\epsilon}^{*(n)}\left(S_{1} \mid \mathbf{s}_{2, b}, \mathbf{w}_{3, b}, \mathbf{t}_{b}\right)\right\}$ via an expression that is independent of $\tilde{\mathbf{s}}_{1}$. To reduce clutter, let us denote $\mathbf{s}_{2, b}$, $\mathbf{t}_{b}, \quad \mathbf{q}\left(\mathbf{t}_{b}\right), \quad \mathbf{v}_{1}\left(u_{1, b-1}\right), \quad \mathbf{v}_{2}\left(u_{2, b-1}\right), \quad \mathbf{x}_{1}\left(\tilde{\mathbf{s}}_{1}, u_{1, b-1}, \mathbf{q}\left(\mathbf{t}_{b}\right)\right)$, $\mathbf{x}_{2}\left(\mathbf{s}_{2, b}, u_{2, b-1}, \mathbf{q}\left(\mathbf{t}_{b}\right)\right), \mathbf{x}_{3}\left(u_{1, b-1}, u_{2, b-1}\right), \mathbf{w}_{3, b}, \mathbf{y}_{3, b}$ by $\mathbf{s}_{2}, \mathbf{t}$, $\mathbf{q}, \mathbf{v}_{1}, \mathbf{v}_{2}, \tilde{\mathbf{x}}_{1}, \mathbf{x}_{2}, \mathbf{x}_{3}, \mathbf{w}_{3}, \mathbf{y}_{3}$, respectively. Note that the joint distribution obeys

$$
\begin{gathered}
p\left(\tilde{\mathbf{s}}_{1}, \mathbf{s}_{2}, \mathbf{t}, \mathbf{q}, \mathbf{v}_{1}, \mathbf{v}_{2}, \tilde{\mathbf{x}}_{1}, \mathbf{x}_{2}, \mathbf{x}_{3}, \mathbf{w}_{3}, \mathbf{y}_{3}\right) \\
=\prod_{j=1}^{n} p\left(s_{2, j}, w_{3, j}, t_{j}\right) p\left(\tilde{s}_{1, j}\right) \times \\
p\left(v_{1, j}, v_{2, j}, x_{3, j}, q_{j} \mid \tilde{s}_{1, j}, s_{2, j}, w_{3, j}, t_{j}\right) \times \\
p\left(x_{2, j}, y_{3, j} \mid s_{2, j}, w_{3, j}, t_{j}, v_{1, j}, v_{2, j}, x_{3, j}, q_{j}\right) \times \\
p\left(\tilde{x}_{1, j} \mid v_{1, j}, v_{2, j}, x_{3, j}, q_{j}, \tilde{s}_{1, j}\right) .
\end{gathered}
$$

Next, we use the assignments

$$
\begin{aligned}
& \mathbf{z}_{1}=\left(\mathbf{s}_{2}, \mathbf{w}_{3}, \mathbf{t}\right), \quad \mathbf{z}_{2}=\tilde{\mathbf{s}}_{1}, \quad \mathbf{Z}_{3}=\left(\mathbf{V}_{1}, \mathbf{V}_{2}, \mathbf{X}_{3}, \mathbf{Q}\right), \\
& \mathbf{Z}_{4}=\tilde{\mathbf{X}}_{1}, \quad \mathbf{Z}_{5}=\left(\mathbf{X}_{2}, \mathbf{Y}_{3}\right) .
\end{aligned}
$$

Equation (B.8) shows that the assignments $(B .9)$ satisfy the assumptions of [7, Lemma, Appendix A]. Using this lemma we bound $\operatorname{Pr}\left\{E_{21}^{r}\left(\tilde{\mathbf{s}}_{1}\right) \mid\left(E_{1}^{r}\right)^{c}, \tilde{\mathbf{s}}_{1} \in A_{\epsilon}^{*(n)}\left(S_{1} \mid \mathbf{s}_{2, b}, \mathbf{w}_{3, b}, \mathbf{t}_{b}\right)\right\}$ as follows

$$
\begin{gathered}
\operatorname{Pr}\left\{E_{21}^{r}\left(\tilde{\mathbf{s}}_{1}\right) \mid\left(E_{1}^{r}\right)^{c}, \tilde{\mathbf{s}}_{1} \in A_{\epsilon}^{*(n)}\left(S_{1} \mid \mathbf{s}_{2, b}, \mathbf{w}_{3, b}, \mathbf{t}_{b}\right)\right\} \\
\leq 2^{-n\left[I\left(X_{1} ; Y_{3} \mid S_{2}, V_{1}, X_{2}, X_{3}, W_{3}, Q\right)-\epsilon_{0}\right]},
\end{gathered}
$$

where $\epsilon_{0}=8 \epsilon$, and we used the fact that $T$ is a function of $S_{2}$, and the Markov chains $V_{2}-\left(S_{2}, V_{1}, X_{2}, X_{3}, W_{3}, Q\right)-Y_{3}$, and $S_{1}-\left(S_{2}, V_{1}, X_{1}, X_{2}, X_{3}, W_{3}, Q\right)-Y_{3}$. Plugging (B.10) into (B.7) we have

$$
\begin{aligned}
\operatorname{Pr}\{ & \left.E_{21}^{r} \mid\left(E_{1}^{r}\right)^{c}\right\} \\
& \leq \sum_{\substack{\tilde{\mathbf{s}}_{1} \neq \mathbf{s}_{1, b}, \tilde{\mathbf{s}}_{1} \in A_{\epsilon}^{*(n)}\left(S_{1} \mid \mathbf{s}_{2}, b \\
\mathbf{w} \\
\mathbf{w}_{3, b}, \mathbf{t}_{b}\right)}} 2^{-n\left[I\left(X_{1} ; Y_{3} \mid S_{2}, V_{1}, X_{2}, X_{3}, W_{3}, Q\right)-\epsilon_{0}\right]} \\
& \leq 2^{n\left[H\left(S_{1} \mid S_{2}, W_{3}, T\right)-I\left(X_{1} ; Y_{3} \mid S_{2}, V_{1}, X_{2}, X_{3}, W_{3}, Q\right)+2 \epsilon_{0}\right]} \\
& =2^{n\left[H\left(S_{1} \mid S_{2}, W_{3}\right)-I\left(X_{1} ; Y_{3} \mid S_{2}, V_{1}, X_{2}, X_{3}, W_{3}, Q\right)+2 \epsilon_{0}\right]},
\end{aligned}
$$

which can be bounded by $\epsilon$, for large enough $n$, as long as

$$
\begin{aligned}
& H\left(S_{1} \mid S_{2}, W_{3}\right) \\
& \quad<I\left(X_{1} ; Y_{3} \mid S_{2}, V_{1}, X_{2}, X_{3}, W_{3}, Q\right)-2 \epsilon_{0} .
\end{aligned}
$$


Following similar arguments as in (B.6)-(B.11), we can also show that $\operatorname{Pr}\left\{E_{22}^{r} \mid\left(E_{1}^{r}\right)^{c}\right\}$ can be bounded by $\epsilon$, for large enough $n$, as long as

$$
\begin{aligned}
& H\left(S_{2} \mid S_{1}, W_{3}\right) \\
& \quad<I\left(X_{2} ; Y_{3} \mid S_{1}, V_{2}, X_{1}, X_{3}, W_{3}, Q\right)-2 \epsilon_{0},
\end{aligned}
$$

and $\operatorname{Pr}\left\{E_{23}^{r} \mid\left(E_{1}^{r}\right)^{c}\right\}$ can be bounded by $\epsilon$, for large enough $n$, as long as

$$
\begin{aligned}
& H\left(S_{1}, S_{2} \mid W_{3}, T\right) \\
& \quad<I\left(X_{1}, X_{2} ; Y_{3} \mid V_{1}, V_{2}, X_{3}, W_{3}, T, Q\right)-2 \epsilon_{0} .
\end{aligned}
$$

To bound $\operatorname{Pr}\left\{E_{24}^{r} \mid\left(E_{1}^{r}\right)^{c}\right\}$ we first define the event $E_{24}^{r}\left(\tilde{\mathbf{s}}_{1}, \tilde{\mathbf{s}}_{2}, \tilde{\mathbf{t}}\right)$ as follows

$$
\begin{aligned}
& E_{24}^{r}\left(\tilde{\mathbf{s}}_{1}, \tilde{\mathbf{s}}_{2}, \tilde{\mathbf{t}}\right) \triangleq \\
& \left\{\mathbf{Q}(\tilde{\mathbf{t}}) \neq \mathbf{Q}\left(\mathbf{t}_{b}\right),\left(\tilde{\mathbf{s}}_{1}, \tilde{\mathbf{s}}_{2}, \tilde{\mathbf{t}}, \mathbf{Q}(\tilde{\mathbf{t}}), \mathbf{V}_{1}\left(u_{1, b-1}\right), \mathbf{V}_{2}\left(u_{2, b-1}\right),\right.\right. \\
& \quad \mathbf{X}_{1}\left(\tilde{\mathbf{s}}_{1}, u_{1, b-1}, \mathbf{Q}(\tilde{\mathbf{t}})\right), \mathbf{X}_{2}\left(\tilde{\mathbf{s}}_{2}, u_{2, b-1}, \mathbf{Q}(\tilde{\mathbf{t}})\right), \\
& \left.\quad \mathbf{X}_{3}\left(u_{1, b-1}, u_{2, b-1}\right), \mathbf{w}_{3, b}, \mathbf{Y}_{3, b} \in A_{\epsilon}^{*(n)}\right\} .
\end{aligned}
$$

Recalling that $\tilde{\mathbf{s}}_{1} \neq \mathbf{s}_{1, b}, \tilde{\mathbf{s}}_{2} \neq \mathbf{s}_{2, b}, \tilde{\mathbf{t}} \neq \mathbf{t}_{b}$, we have

$$
\begin{aligned}
& \operatorname{Pr}\left\{E_{24}^{r} \mid\left(E_{1}^{r}\right)^{c}\right\} \\
& \leq \quad \sum_{\tilde{\mathbf{s}}_{1} \neq \mathbf{s}_{1, b}, \tilde{\mathbf{s}}_{2} \neq \mathbf{s}_{2, b}, \tilde{\mathbf{t}} \neq \mathbf{t}_{b},} \operatorname{Pr}_{\left(\mathbf{s}_{1}\right.}\left\{E_{24}^{r}\left(\tilde{\mathbf{s}}_{1}, \tilde{\mathbf{s}}_{2}, \tilde{\mathbf{t}}\right) \mid\left(E_{1}^{r}\right)^{c}\right\} . \\
& \left(\tilde{\mathbf{s}}_{1}, \tilde{\mathbf{s}}_{2}, \tilde{\mathbf{t}}\right) \in A_{\epsilon}^{*(n)}\left(S_{1}, S_{2}, T \mid \mathbf{w}_{3, b}\right)
\end{aligned}
$$

Let $\mathscr{A}_{b}$ denote the event that $\left(\tilde{\mathbf{s}}_{1}, \tilde{\mathbf{s}}_{2}, \tilde{\mathbf{t}}\right) \in$ $A_{\epsilon}^{*(n)}\left(S_{1}, S_{2}, T \mid \mathbf{w}_{3, b}\right)$. Note that if $\mathscr{A}_{b}^{c}$ holds then $\operatorname{Pr}\left\{E_{24}^{r}\left(\tilde{\mathbf{s}}_{1}, \tilde{\mathbf{s}}_{2}, \tilde{\mathbf{t}}\right) \mid\left(E_{1}^{r}\right)^{c}, \mathscr{A}_{b}^{c}\right\}=0$. Hence, we can write (B.17) at the bottom of the page, where B.17) follows from [37, Thm. 6.7]. In the following we upper bound the summands in (B.17) independently of $\tilde{\mathbf{s}}_{1}, \tilde{\mathbf{s}}_{2}, \tilde{\mathbf{t}}$, and $\tilde{\mathbf{q}}$. To reduce clutter let us denote $\mathbf{v}_{1}\left(u_{1, b-1}\right), \mathbf{v}_{2}\left(u_{2, b-1}\right)$, $\mathbf{x}_{1}\left(\tilde{\mathbf{s}}_{1}, u_{1, b-1}, \tilde{\mathbf{q}}\right), \mathbf{x}_{2}\left(\tilde{\mathbf{s}}_{2}, u_{2, b-1}, \tilde{\mathbf{q}}\right), \mathbf{x}_{3}\left(u_{1, b-1}, u_{2, b-1}\right), \mathbf{w}_{3, b}$, $\mathbf{y}_{3, b}$ by $\mathbf{v}_{1}, \mathbf{v}_{2}, \tilde{\mathbf{x}}_{1}, \tilde{\mathbf{x}}_{2}, \mathbf{x}_{3}, \mathbf{w}_{3}, \mathbf{y}_{3}$, respectively. The joint distribution obeys

$$
\begin{aligned}
& p\left(\tilde{\mathbf{s}}_{1}, \tilde{\mathbf{s}}_{2}, \tilde{\mathbf{t}}, \tilde{\mathbf{q}}, \mathbf{v}_{1}, \mathbf{v}_{2}, \tilde{\mathbf{x}}_{1}, \tilde{\mathbf{x}}_{2}, \mathbf{x}_{3}, \mathbf{w}_{3}, \mathbf{y}_{3}\right) \\
& =\prod_{j=1}^{n} p\left(w_{3, j}\right) p\left(\tilde{s}_{1, j}, \tilde{s}_{2, j}, \tilde{t}_{j}, \tilde{q}_{j}\right) \times \\
& \quad p\left(v_{1, j}, v_{2, j}, x_{3, j} \mid \tilde{s}_{1, j}, \tilde{s}_{2, j}, w_{3, j}, \tilde{t}_{j}, \tilde{q}_{j}\right) \times \\
& p\left(\tilde{x}_{1, j}, \tilde{x}_{2, j} \mid \tilde{s}_{1, j}, \tilde{s}_{2, j}, \tilde{t}_{j}, \tilde{q}_{j}, v_{1, j}, v_{2, j}, x_{3, j}\right) \times \\
& p\left(y_{3, j} \mid w_{3, j}, v_{1, j}, v_{2, j}, x_{3, j}\right) .
\end{aligned}
$$

Moreover, note that the independence of $Q$ from $\left(S_{1}, S_{2}, T\right)$, and conditioning on $\mathscr{A}_{b}$, implies that for $n$ large enough $\left(\tilde{\mathbf{s}}_{1}, \tilde{\mathbf{s}}_{2}, \tilde{\mathbf{t}}, \tilde{\mathbf{q}}\right) \in A_{\epsilon_{2}}^{*(n)}\left(S_{1}, S_{2}, T, Q \mid \mathbf{w}_{3, b}\right)$. Hence, we can use [7. Lemma, Appendix A] with the following assignments:

$$
\begin{aligned}
& \mathbf{z}_{1}=\mathbf{w}_{3}, \quad \mathbf{z}_{2}=\left(\tilde{\mathbf{s}}_{1}, \tilde{\mathbf{s}}_{2}, \tilde{\mathbf{t}}, \tilde{\mathbf{q}}\right), \quad \mathbf{Z}_{3}=\left(\mathbf{X}_{3}, \mathbf{V}_{1}, \mathbf{V}_{2}\right), \\
& \mathbf{Z}_{4}=\left(\tilde{\mathbf{X}}_{1}, \tilde{\mathbf{X}}_{2}\right), \quad \mathbf{Z}_{5}=\mathbf{Y}_{3},
\end{aligned}
$$

to bound

$$
\begin{aligned}
\operatorname{Pr}\left\{\left(\tilde{\mathbf{s}}_{1}, \tilde{\mathbf{s}}_{2}, \tilde{\mathbf{t}}, \tilde{\mathbf{q}}, \mathbf{V}_{1}\left(u_{1, b-1}\right), \mathbf{V}_{2}\left(u_{2, b-1}\right), \mathbf{X}_{1}\left(\tilde{\mathbf{s}}_{1}, u_{1, b-1}, \tilde{\mathbf{q}}\right),\right.\right. \\
\left.\quad \mathbf{X}_{2}\left(\tilde{\mathbf{s}}_{2}, u_{2, b-1}, \tilde{\mathbf{q}}\right), \mathbf{X}_{3}\left(u_{1, b-1}, u_{2, b-1}\right), \mathbf{w}_{3, b}, \mathbf{Y}_{3, b}\right) \\
\in A_{\epsilon_{2}}^{*(n)} \mid\left(E_{1}^{r}\right)^{c}, \mathbf{Q}\left(\mathbf{t}_{b}\right) \neq \tilde{\mathbf{q}}, \\
\left.\left(\tilde{\mathbf{s}}_{1}, \tilde{\mathbf{s}}_{2}, \tilde{\mathbf{t}}, \tilde{\mathbf{q}}\right) \in A_{\epsilon_{2}}^{*(n)}\left(S_{1}, S_{2}, T, Q \mid \mathbf{w}_{3, b}\right)\right\} \\
\quad \leq 2^{-n\left[I\left(S_{1}, S_{2}, T, Q, X_{1}, X_{2} ; Y_{3} \mid V_{1}, V_{2}, X_{3}, W_{3}\right)-\epsilon_{0}\right]} \\
\quad \stackrel{(a)}{=} 2^{-n\left[I\left(X_{1}, X_{2} ; Y_{3} \mid V_{1}, V_{2}, X_{3}, W_{3}\right)-\epsilon_{0}\right]},
\end{aligned}
$$

where $(a)$ follows from the Markov chain $\left(S_{1}, S_{2}, T, Q\right)-$ $\left(X_{1}, X_{2}, V_{1}, V_{2}, X_{3}, W_{3}\right)-Y_{3}$. From (B.20) and (B.17) we obtain

$$
\begin{aligned}
& \operatorname{Pr}\left\{E_{24}^{r}\left(\tilde{\mathbf{s}}_{1}, \tilde{\mathbf{s}}_{2}, \tilde{\mathbf{t}}\right) \mid \mathscr{A}_{b},\left(E_{1}^{r}\right)^{c}\right\} \\
& \quad \leq \sum_{\tilde{\mathbf{q}} \in A_{\epsilon}^{*(n)}(Q)} 2^{-n\left[I\left(X_{1}, X_{2} ; Y_{3} \mid V_{1}, V_{2}, X_{3}, W_{3}\right)-\epsilon_{0}\right]} 2^{-n\left[H(Q)-\epsilon_{1}\right]},
\end{aligned}
$$

and by using the bound $\left|A_{\epsilon}^{*(n)}(Q)\right| \leq 2^{n\left[H(Q)+\epsilon_{1}\right]}$, [37, Thm. 6.2 , we have that

$$
\begin{aligned}
\operatorname{Pr} & \left\{E_{24}^{r}\left(\tilde{\mathbf{s}}_{1}, \tilde{\mathbf{s}}_{2}, \tilde{\mathbf{t}}\right) \mid \mathscr{A}_{b},\left(E_{1}^{r}\right)^{c}\right\} \\
& \leq 2^{-n\left[I\left(X_{1}, X_{2} ; Y_{3} \mid V_{1}, V_{2}, X_{3}, W_{3}\right)-\epsilon_{0}-2 \epsilon_{1}\right]} .
\end{aligned}
$$

$$
\begin{aligned}
& \operatorname{Pr}\left\{E_{24}^{r}\left(\tilde{\mathbf{s}}_{1}, \tilde{\mathbf{s}}_{2}, \tilde{\mathbf{t}}\right) \mid \mathscr{A}_{b},\left(E_{1}^{r}\right)^{c}\right\} \\
& \leq \sum_{\tilde{\mathbf{q}} \in \mathcal{Q}^{n}} \operatorname{Pr}\left\{\mathbf{Q}(\tilde{\mathbf{t}})=\tilde{\mathbf{q}} \mid \mathscr{A}_{b},\left(E_{1}^{r}\right)^{c}\right\} \operatorname{Pr}\left\{\mathbf{Q}\left(\mathbf{t}_{b}\right) \neq \tilde{\mathbf{q}} \mid \mathscr{A}_{b},\left(E_{1}^{r}\right)^{c}\right\} \times \\
& \operatorname{Pr}\left\{\left(\tilde{\mathbf{s}}_{1}, \tilde{\mathbf{s}}_{2}, \tilde{\mathbf{t}}, \tilde{\mathbf{q}}, \mathbf{V}_{1}\left(u_{1, b-1}\right), \mathbf{V}_{2}\left(u_{2, b-1}\right), \mathbf{X}_{1}\left(\tilde{\mathbf{s}}_{1}, u_{1, b-1}, \tilde{\mathbf{q}}\right), \mathbf{X}_{2}\left(\tilde{\mathbf{s}}_{2}, u_{2, b-1}, \tilde{\mathbf{q}}\right),\right.\right. \\
& \left.\left.\quad \mathbf{X}_{3}\left(u_{1, b-1}, u_{2, b-1}\right), \mathbf{w}_{3, b}, \mathbf{Y}_{3, b}\right) \in A_{\epsilon}^{*(n)} \mid \mathscr{A}_{b},\left(E_{1}^{r}\right)^{c}, \mathbf{Q}\left(\mathbf{t}_{b}\right) \neq \tilde{\mathbf{q}}\right\} \\
& \leq \sum_{\tilde{\mathbf{q}} \in A_{\epsilon}^{*(n)}(Q)} \operatorname{Pr}\left\{\mathbf{Q}(\tilde{\mathbf{t}})=\tilde{\mathbf{q}} \mid \mathscr{A}_{b},\left(E_{1}^{r}\right)^{c}\right\} \operatorname{Pr}\left\{\left(\tilde{\mathbf{s}}_{1}, \tilde{\mathbf{s}}_{2}, \tilde{\mathbf{t}}, \tilde{\mathbf{q}}, \mathbf{V}_{1}\left(u_{1, b-1}\right), \mathbf{V}_{2}\left(u_{2, b-1}\right), \mathbf{X}_{1}\left(\tilde{\mathbf{s}}_{1}, u_{1, b-1}, \tilde{\mathbf{q}}\right),\right.\right. \\
& \left.\left.\mathbf{X}_{2}\left(\tilde{\mathbf{s}}_{2}, u_{2, b-1}, \tilde{\mathbf{q}}\right), \mathbf{X}_{3}\left(u_{1, b-1}, u_{2, b-1}\right), \mathbf{w}_{3, b}, \mathbf{Y}_{3, b}\right) \in A_{\epsilon}^{*(n)} \mid \mathscr{A}_{b},\left(E_{1}^{r}\right)^{c}, \mathbf{Q}\left(\mathbf{t}_{b}\right) \neq \tilde{\mathbf{q}}\right\},
\end{aligned}
$$


Plugging (B.22) into (B.16) we have

$$
\begin{aligned}
\operatorname{Pr}\{ & \left.E_{24}^{r} \mid\left(E_{1}^{r}\right)^{c}\right\} \\
& \leq\left|A_{\epsilon}^{*(n)}\left(S_{1}, S_{2}, T \mid \mathbf{w}_{3, b}\right)\right| \times \\
& 2^{-n\left[I\left(X_{1}, X_{2} ; Y_{3} \mid V_{1}, V_{2}, X_{3}, W_{3}\right)-\epsilon_{0}-2 \epsilon_{1}\right]} \\
& \leq 2^{n\left[H\left(S_{1}, S_{2} \mid W_{3}\right)-I\left(X_{1}, X_{2} ; Y_{3} \mid V_{1}, V_{2}, X_{3}, W_{3}\right)+4 \epsilon_{0}\right]},
\end{aligned}
$$

which can be bounded by $\epsilon$, for large enough $n$, if $H\left(S_{1}, S_{2} \mid W_{3}\right)<I\left(X_{1}, X_{2} ; Y_{3} \mid V_{1}, V_{2}, X_{3}, W_{3}\right)-4 \epsilon_{0}$.

Lastly, to bound $\operatorname{Pr}\left\{E_{25}^{r} \mid\left(E_{1}^{r}\right)^{c}\right\}$ we first define the event $E_{25}^{r}\left(\tilde{\mathbf{s}}_{1}, \tilde{\mathbf{s}}_{2}, \tilde{\mathbf{t}}\right)$ as follows

$$
\begin{aligned}
& E_{25}^{r}\left(\tilde{\mathbf{s}}_{1}, \tilde{\mathbf{s}}_{2}, \tilde{\mathbf{t}}\right) \triangleq \\
& \left\{\mathbf{Q}(\tilde{\mathbf{t}})=\mathbf{Q}\left(\mathbf{t}_{b}\right),\left(\tilde{\mathbf{s}}_{1}, \tilde{\mathbf{s}}_{2}, \tilde{\mathbf{t}}, \mathbf{Q}(\tilde{\mathbf{t}}), \mathbf{V}_{1}\left(u_{1, b-1}\right), \mathbf{V}_{2}\left(u_{2, b-1}\right),\right.\right. \\
& \quad \mathbf{X}_{1}\left(\tilde{\mathbf{s}}_{1}, u_{1, b-1}, \mathbf{Q}(\tilde{\mathbf{t}})\right), \mathbf{X}_{2}\left(\tilde{\mathbf{s}}_{2}, u_{2, b-1}, \mathbf{Q}(\tilde{\mathbf{t}})\right), \\
& \left.\left.\quad \mathbf{X}_{3}\left(u_{1, b-1}, u_{2, b-1}\right), \mathbf{w}_{3, b}, \mathbf{Y}_{3, b}\right) \in A_{\epsilon}^{*(n)}\right\} .
\end{aligned}
$$

Recalling that $\tilde{\mathbf{s}}_{1} \neq \mathbf{s}_{1, b}, \tilde{\mathbf{s}}_{2} \neq \mathbf{s}_{2, b}, \tilde{\mathbf{t}} \neq \mathbf{t}_{b}$, we have

$$
\begin{aligned}
& \operatorname{Pr}\left\{E_{25}^{r} \mid\left(E_{1}^{r}\right)^{c}\right\} \\
& \leq \sum_{\substack{\tilde{\mathbf{s}}_{1} \neq \mathbf{s}_{1, b}, \tilde{\mathbf{s}}_{2} \neq \mathbf{s}_{2, b}, \tilde{\mathbf{t}} \neq \mathbf{t}_{b},\left(\tilde{\mathbf{s}}_{1}, \tilde{\mathbf{s}}_{2}, \tilde{\mathbf{t}}\right) \in A_{\epsilon}^{*(n)}\left(S_{1}, S_{2}, T \mid \mathbf{w}_{3, b}\right)}} \operatorname{Pr}\left\{E_{25}^{r}\left(\tilde{\mathbf{s}}_{1}, \tilde{\mathbf{s}}_{2}, \tilde{\mathbf{t}}\right) \mid\left(E_{1}^{r}\right)^{c}\right\} .
\end{aligned}
$$

Then we have

$$
\begin{gathered}
\operatorname{Pr}\left\{E_{25}^{r}\left(\tilde{\mathbf{s}}_{1}, \tilde{\mathbf{s}}_{2}, \tilde{\mathbf{t}}\right) \mid \mathscr{A}_{b},\left(E_{1}^{r}\right)^{c}\right\} \\
=\sum_{\overline{\mathbf{q}} \in A_{\epsilon}^{*(n)}(Q)} \operatorname{Pr}\left\{\mathbf{Q}(\tilde{\mathbf{t}})=\overline{\mathbf{q}} \mid \mathscr{A}_{b},\left(E_{1}^{r}\right)^{c}\right\} \times \\
\operatorname{Pr}\left\{\mathbf{Q}\left(\mathbf{t}_{b}\right)=\overline{\mathbf{q}} \mid \mathscr{A}_{b},\left(E_{1}^{r}\right)^{c}\right\} \times \\
\operatorname{Pr}\left\{\left(\tilde{\mathbf{s}}_{1}, \tilde{\mathbf{s}}_{2}, \tilde{\mathbf{t}}, \overline{\mathbf{q}}, \mathbf{V}_{1}\left(u_{1, b-1}\right), \mathbf{V}_{2}\left(u_{2, b-1}\right),\right.\right. \\
\mathbf{X}_{1}\left(\tilde{\mathbf{s}}_{1}, u_{1, b-1}, \overline{\mathbf{q}}\right), \mathbf{X}_{2}\left(\tilde{\mathbf{s}}_{2}, u_{2, b-1}, \overline{\mathbf{q}}\right), \\
\left.\mathbf{X}_{3}\left(u_{1, b-1}, u_{2, b-1}\right), \mathbf{w}_{3, b}, \mathbf{Y}_{3, b}\right) \\
\left.\in A_{\epsilon}^{*(n)} \mid \mathscr{A}_{b},\left(E_{1}^{r}\right)^{c}, \mathbf{Q}\left(\mathbf{t}_{b}\right)=\overline{\mathbf{q}}\right\},
\end{gathered}
$$

where B.27 follows from the same argument leading to B.17). In the following we upper bound the summands in B.27 independently of $\tilde{\mathbf{s}}_{1}, \tilde{\mathbf{s}}_{2}, \tilde{\mathbf{t}}$, and $\overline{\mathbf{q}}$. Let us denote $\mathbf{v}_{1}\left(u_{1, b-1}\right), \mathbf{v}_{2}\left(u_{2, b-1}\right), \mathbf{x}_{1}\left(\tilde{\mathbf{s}}_{1}, u_{1, b-1}, \overline{\mathbf{q}}\right), \mathbf{x}_{2}\left(\tilde{\mathbf{s}}_{2}, u_{2, b-1}, \overline{\mathbf{q}}\right)$, $\mathbf{x}_{3}\left(u_{1, b-1}, u_{2, b-1}\right), \mathbf{w}_{3, b}, \mathbf{y}_{3, b}$ by $\mathbf{v}_{1}, \mathbf{v}_{2}, \tilde{\mathbf{x}}_{1}, \tilde{\mathbf{x}}_{2}, \mathbf{x}_{3}, \mathbf{w}_{3}, \mathbf{y}_{3}$, respectively. Note that the joint distribution obeys

$$
\begin{gathered}
p\left(\tilde{\mathbf{s}}_{1}, \tilde{\mathbf{s}}_{2}, \tilde{\mathbf{t}}, \overline{\mathbf{q}}, \mathbf{v}_{1}, \mathbf{v}_{2}, \tilde{\mathbf{x}}_{1}, \tilde{\mathbf{x}}_{2}, \mathbf{x}_{3}, \mathbf{w}_{3}, \mathbf{y}_{3}\right) \\
=\prod_{j=1}^{n} p\left(\tilde{s}_{1, j}, \tilde{s}_{2, j}, \tilde{t}_{j}, \bar{q}_{j}, w_{3, j}\right) \times \\
p\left(v_{1, j}, v_{2, j}, x_{3, j} \mid \tilde{s}_{1, j}, \tilde{s}_{2, j}, w_{3, j}, \tilde{t}_{j}, \bar{q}_{j}\right) \times \\
p\left(\tilde{x}_{1, j}, \tilde{x}_{2, j} \mid \tilde{s}_{1, j}, \tilde{s}_{2, j}, \tilde{t}_{j}, \bar{q}_{j}, v_{1, j}, v_{2, j}, x_{3, j}\right) \times \\
p\left(y_{3, j} \mid w_{3, j}, v_{1, j}, v_{2, j}, x_{3, j}, \bar{q}_{j}\right) .
\end{gathered}
$$

Similarly to the analysis for $E_{24}^{r}\left(\tilde{\mathbf{s}}_{1}, \tilde{\mathbf{s}}_{2}, \tilde{\mathbf{t}}\right)$ we have that $\left(\tilde{\mathbf{s}}_{1}, \tilde{\mathbf{s}}_{2}, \tilde{\mathbf{t}}, \overline{\mathbf{q}}\right) \in A_{\epsilon_{2}}^{*(n)}\left(S_{1}, S_{2}, T, Q \mid \mathbf{w}_{3, b}\right)$. Hence, we can use [7. Lemma, Appendix A] with the following assignments:

$$
\begin{aligned}
& \mathbf{z}_{1}=\left(\mathbf{w}_{3}, \overline{\mathbf{q}}\right), \quad \mathbf{z}_{2}=\left(\tilde{\mathbf{s}}_{1}, \tilde{\mathbf{s}}_{2}, \tilde{\mathbf{t}}, \overline{\mathbf{q}}\right), \quad \mathbf{Z}_{3}=\left(\mathbf{X}_{3}, \mathbf{V}_{1}, \mathbf{V}_{2}\right), \\
& \mathbf{Z}_{4}=\left(\tilde{\mathbf{X}}_{1}, \tilde{\mathbf{X}}_{2}\right), \quad \mathbf{Z}_{5}=\mathbf{Y}_{3} .
\end{aligned}
$$

Then we get the following bound:

$$
\begin{gathered}
\operatorname{Pr}\left\{\left(\tilde{\mathbf{s}}_{1}, \tilde{\mathbf{s}}_{2}, \tilde{\mathbf{t}}, \overline{\mathbf{q}}, \mathbf{V}_{1}\left(u_{1, b-1}\right), \mathbf{V}_{2}\left(u_{2, b-1}\right), \mathbf{X}_{1}\left(\tilde{\mathbf{s}}_{1}, u_{1, b-1}, \overline{\mathbf{q}}\right),\right.\right. \\
\left.\mathbf{X}_{2}\left(\tilde{\mathbf{s}}_{2}, u_{2, b-1}, \overline{\mathbf{q}}\right), \mathbf{X}_{3}\left(u_{1, b-1}, u_{2, b-1}\right), \mathbf{w}_{3, b}, \mathbf{Y}_{3, b}\right) \\
\in A_{\epsilon_{2}}^{*(n)} \mid\left(E_{1}^{r}\right)^{c}, \mathbf{Q}\left(\mathbf{t}_{b}\right)=\overline{\mathbf{q}}, \\
\left.\left(\tilde{\mathbf{s}}_{1}, \tilde{\mathbf{s}}_{2}, \tilde{\mathbf{t}}, \overline{\mathbf{q}}\right) \in A_{\epsilon_{2}}^{*(n)}\left(S_{1}, S_{2}, T, Q \mid \mathbf{w}_{3, b}\right)\right\} \\
\stackrel{(a)}{\leq} 2^{-n\left[I\left(X_{1}, X_{2} ; Y_{3} \mid V_{1}, V_{2}, X_{3}, W_{3}, Q\right)-\epsilon_{0}\right]},
\end{gathered}
$$

where $(a)$ follows from the Markov chain $\left(S_{1}, S_{2}, T\right)-$ $\left(X_{1}, X_{2}, V_{1}, V_{2}, X_{3}, W_{3}\right)-Y_{3}$. From (B.30) and B.27) we have

$$
\begin{aligned}
& \operatorname{Pr}\left\{E_{25}^{r}\left(\tilde{\mathbf{s}}_{1}, \tilde{\mathbf{s}}_{2}, \tilde{\mathbf{t}}\right) \mid \mathscr{A}_{b},\left(E_{1}^{r}\right)^{c}\right\} \\
& \leq \sum_{\overline{\mathbf{q}} \in A_{\epsilon}^{*(n)}(Q)} 2^{-n\left[I\left(X_{1}, X_{2} ; Y_{3} \mid V_{1}, V_{2}, X_{3}, W_{3}, Q\right)-\epsilon_{0}\right]} \times \\
& \operatorname{Pr}\left\{\mathbf{Q}(\tilde{\mathbf{t}})=\overline{\mathbf{q}} \mid \mathscr{A}_{b},\left(E_{1}^{r}\right)^{c}\right\} \times \\
& \operatorname{Pr}\left\{\mathbf{Q}\left(\mathbf{t}_{b}\right)=\overline{\mathbf{q}} \mid \mathscr{A}_{b},\left(E_{1}^{r}\right)^{c}\right\} .
\end{aligned}
$$

However, for $\overline{\mathbf{q}} \in A_{\epsilon}^{*(n)}(Q)$ the following holds

$$
\begin{gathered}
\operatorname{Pr}\left\{\mathbf{Q}(\tilde{\mathbf{t}})=\overline{\mathbf{q}} \mid \mathscr{A}_{b},\left(E_{1}^{r}\right)^{c}\right\} \leq 2^{-n\left[H(Q)-\epsilon_{1}\right]}, \\
\operatorname{Pr}\left\{\mathbf{Q}\left(\mathbf{t}_{b}\right)=\overline{\mathbf{q}} \mid \mathscr{A}_{b},\left(E_{1}^{r}\right)^{c}\right\} \leq 2^{-n\left[H(Q)-\epsilon_{1}\right]} .
\end{gathered}
$$

Hence, using the fact that $\left|A_{\epsilon}^{*(n)}(Q)\right| \leq 2^{n\left[H(Q)+\epsilon_{1}\right]}$, we have that

$$
\begin{aligned}
\operatorname{Pr}\{ & \left.E_{25}^{r}\left(\tilde{\mathbf{s}}_{1}, \tilde{\mathbf{s}}_{2}, \tilde{\mathbf{t}}\right) \mid \mathscr{A}_{b},\left(E_{1}^{r}\right)^{c}\right\} \\
& \leq 2^{-n\left[I\left(X_{1}, X_{2} ; Y_{3} \mid V_{1}, V_{2}, X_{3}, W_{3}, Q\right)+H(Q)-\epsilon_{0}-3 \epsilon_{1}\right]} .
\end{aligned}
$$

Finally, plugging (B.32) into B.26 we have

$$
\begin{aligned}
\operatorname{Pr} & \left\{E_{25}^{r} \mid\left(E_{1}^{r}\right)^{c}\right\} \\
& \leq\left|A_{\epsilon}^{*(n)}\left(S_{1}, S_{2}, T \mid \mathbf{w}_{3, b}\right)\right| \times \\
& \quad 2^{-n\left[I\left(X_{1}, X_{2} ; Y_{3} \mid V_{1}, V_{2}, X_{3}, W_{3}, Q\right)+H(Q)-\epsilon_{0}-3 \epsilon_{1}\right]} \\
& \leq 2^{n\left[H\left(S_{1}, S_{2} \mid W_{3}\right)-I\left(X_{1}, X_{2} ; Y_{3} \mid V_{1}, V_{2}, X_{3}, W_{3}, Q\right)-H(Q)+5 \epsilon_{0}\right]},
\end{aligned}
$$

which can be bounded by $\epsilon$, for large enough $n$, as long as

$$
\begin{aligned}
& H\left(S_{1}, S_{2} \mid W_{3}\right) \\
& \quad<I\left(X_{1}, X_{2} ; Y_{3} \mid V_{1}, V_{2}, X_{3}, W_{3}, Q\right)+H(Q)-5 \epsilon_{0} .
\end{aligned}
$$

However, condition B.34 is redundant since it is dominated by condition (B.24), hence, we conclude that if conditions (30a)-30d hold, then for large enough $n$,

$$
\operatorname{Pr}\left\{E_{2}^{r} \mid\left(E_{1}^{r}\right)^{c}\right\} \leq \sum_{j=1}^{5} \operatorname{Pr}\left\{E_{2 j}^{r} \mid\left(E_{1}^{r}\right)^{c}\right\} \leq 5 \epsilon .
$$

Combining equations (B.4), B.5 and (B.35) yields

$$
\bar{P}_{r}^{(n)} \leq \operatorname{Pr}\left\{E_{2}^{r} \mid\left(E_{1}^{r}\right)^{c}\right\}+2 \epsilon \leq 7 \epsilon .
$$

Next the destination error probability analysis is derived.

Channel decoder: Let $E_{c h}^{d}\left(u_{1, b}, u_{2, b} ; \mathbf{s}_{1, b+1}, \mathbf{s}_{2, b+1}\right)$ denote the channel decoding error event for decoding $\left(u_{1, b}, u_{2, b}\right)$ 
at the destination at block $b$, assuming $\left(\mathbf{s}_{1, b+1}, \mathbf{s}_{2, b+1}\right)$ is available at the destination, namely, the event that $\left(\hat{u}_{1, b}, \hat{u}_{2, b}\right) \neq$ $\left(u_{1, b}, u_{2, b}\right)$. Let $\mathbf{t}_{b+1}=h_{1}\left(\mathbf{s}_{1, b+1}\right)=h_{2}\left(\mathbf{s}_{2, b+1}\right)$. The average probability of channel decoding error at the destination at block $b, \bar{P}_{d, c h}^{(n)}$, is defined in B.37 at the bottom of the page, where in step (a) leading to B.37 we apply similar reasoning as [7, Eq. (16)]. In the following we show that the inner sum in B.37) can be upper bounded independently of $\left(\mathbf{s}_{1, b+1}, \mathbf{s}_{2, b+1}\right)$. Assuming correct decoding at block $b+1$ (hence $\left(\mathbf{s}_{1, b+1}, \mathbf{s}_{2, b+1}\right)$ are available at the destination), we now define the following events:

$$
\begin{gathered}
E_{1}^{d \triangleq\{}\left(\mathbf{s}_{1, b+1}, \mathbf{s}_{2, b+1}, \mathbf{t}_{b+1}, \mathbf{Q}\left(\mathbf{t}_{b+1}\right),\right. \\
\mathbf{V}_{1}\left(u_{1, b}\right), \mathbf{V}_{2}\left(u_{2, b}\right), \mathbf{X}_{1}\left(\mathbf{s}_{1, b+1}, u_{1, b}, \mathbf{Q}\left(\mathbf{t}_{b+1}\right)\right), \\
\mathbf{X}_{2}\left(\mathbf{s}_{2, b+1}, u_{2, b}, \mathbf{Q}\left(\mathbf{t}_{b+1}\right)\right), \mathbf{X}_{3}\left(u_{1, b}, u_{2, b}\right), \\
\left.\left.\mathbf{w}_{b+1}, \mathbf{Y}_{b+1}\right) \notin A_{\epsilon}^{*(n)}\right\}, \\
E_{2}^{d} \triangleq\left\{\hat{u}_{1} \in \mathcal{U}_{1}: \hat{u}_{1} \neq u_{1, b},\right. \\
\left(\mathbf{s}_{1, b+1}, \mathbf{s}_{2, b+1}, \mathbf{t}_{b+1}, \mathbf{Q}\left(\mathbf{t}_{b+1}\right),\right. \\
\mathbf{V}_{1}\left(\hat{u}_{1}\right), \mathbf{V}_{2}\left(u_{2, b}\right), \mathbf{X}_{1}\left(\mathbf{s}_{1, b+1}, \hat{u}_{1}, \mathbf{Q}\left(\mathbf{t}_{b+1}\right)\right), \\
\mathbf{X}_{2}\left(\mathbf{s}_{2, b+1}, u_{2, b}, \mathbf{Q}\left(\mathbf{t}_{b+1}\right)\right), \mathbf{X}_{3}\left(\hat{u}_{1}, u_{2, b}\right), \\
\left.\left.\mathbf{w}_{b+1}, \mathbf{Y}_{b+1}\right) \in A_{\epsilon}^{*}(n)\right\}, \\
E_{3}^{d \triangleq\{}\left\{\hat{u}_{2} \in \mathcal{U}_{2}: \hat{u}_{2} \neq u_{2, b},\right. \\
\left(\mathbf{s}_{1, b+1}, \mathbf{s}_{2, b+1}, \mathbf{t}_{b+1}, \mathbf{Q}\left(\mathbf{t}_{b+1}\right),\right. \\
\mathbf{V}_{1}\left(u_{1, b}\right), \mathbf{V}_{2}\left(\hat{u}_{2}\right), \mathbf{X}_{1}\left(\mathbf{s}_{1, b+1}, u_{1, b}, \mathbf{Q}\left(\mathbf{t}_{b+1}\right)\right), \\
\mathbf{X}_{2}\left(\mathbf{s}_{2, b+1}, \hat{u}_{2}, \mathbf{Q}\left(\mathbf{t}_{b+1}\right)\right), \mathbf{X}_{3}\left(u_{1, b}, \hat{u}_{2}\right), \\
\left.\left.\mathbf{w}_{b+1}, \mathbf{Y}_{b+1}\right) \in A_{\epsilon}^{*(n)}\right\}, \\
E_{4}^{d} \triangleq\left\{\exists\left(\hat{u}_{1}, \hat{u}_{2}\right) \in \mathcal{U}_{1} \times \mathcal{U}_{2}: \hat{u}_{1} \neq u_{1, b}, \hat{u}_{2} \neq u_{2, b},\right. \\
\left(\mathbf{s}_{1, b+1}, \mathbf{s}_{2, b+1}, \mathbf{t}_{b+1}, \mathbf{Q}\left(\mathbf{t}_{b+1}\right),\right. \\
\mathbf{V}_{1}\left(\hat{u}_{1}\right), \mathbf{V}_{2}\left(\hat{u}_{2}\right), \mathbf{X}_{1}\left(\mathbf{s}_{1, b+1}, \hat{u}_{1}, \mathbf{Q}\left(\mathbf{t}_{b+1}\right)\right), \\
\mathbf{X}_{2}\left(\mathbf{s}_{2, b+1}, \hat{u}_{2}, \mathbf{Q}\left(\mathbf{t}_{b+1}\right)\right), \mathbf{X}_{3}\left(\hat{u}_{1}, \hat{u}_{2}\right), \\
\left.\left.\mathbf{w}_{b+1}, \mathbf{Y}_{b+1}\right) \in A_{\epsilon}^{*}(n)\right\} .
\end{gathered}
$$

The average probability of error for decoding $\left(u_{1, b}, u_{2, b}\right)$ at the destination at block $b$, for fixed $\left(\mathbf{s}_{1, b+1}, \mathbf{s}_{2, b+1}\right)$, subject to the event $\mathscr{D}_{b+1}$, is then upper bounded by

$$
\begin{aligned}
& \operatorname{Pr}\left\{E_{c h}^{d}\left(u_{1, b}, u_{2, b} ; \mathbf{s}_{1, b+1}, \mathbf{s}_{2, b+1}\right) \mid \mathscr{D}_{b+1}\right\} \\
& \leq \operatorname{Pr}\left\{E_{1}^{d} \mid \mathscr{D}_{b+1}\right\}+\sum_{j=2}^{4} \operatorname{Pr}\left\{E_{j}^{d} \mid\left(E_{1}^{d}\right)^{c}\right\},
\end{aligned}
$$

where $\mathrm{B} .38$ follows from the union bound. From the AEP [37. Ch. 5.1], for sufficiently large $n, \operatorname{Pr}\left\{E_{1}^{d} \mid \mathscr{D}_{b+1}\right\}$ can be upper bounded by $\epsilon$ for $n$ large enough. Let $\epsilon_{0}$ be a positive number such that $\epsilon_{0}>\epsilon$ and $\epsilon_{0} \rightarrow 0$ as $\epsilon \rightarrow 0$. To bound $\operatorname{Pr}\left\{E_{2}^{d}\left(\hat{u}_{1}\right) \mid\left(E_{1}^{d}\right)^{c}\right\}$ we first define the event $E_{2}^{d}\left(\hat{u}_{1}\right)$ as follows

$$
\begin{aligned}
& E_{2}^{d}\left(\hat{u}_{1}\right) \triangleq \\
& \left\{\left(\mathbf{s}_{1, b+1}, \mathbf{s}_{2, b+1}, \mathbf{t}_{b+1}, \mathbf{Q}\left(\mathbf{t}_{b+1}\right), \mathbf{V}_{1}\left(\hat{u}_{1}\right), \mathbf{V}_{2}\left(u_{2, b}\right),\right.\right. \\
& \quad \mathbf{X}_{1}\left(\mathbf{s}_{1, b+1}, \hat{u}_{1}, \mathbf{Q}\left(\mathbf{t}_{b+1}\right)\right), \mathbf{X}_{2}\left(\mathbf{s}_{2, b+1}, u_{2, b}, \mathbf{Q}\left(\mathbf{t}_{b+1}\right)\right), \\
& \left.\left.\quad \mathbf{X}_{3}\left(\hat{u}_{1}, u_{2, b}\right), \mathbf{w}_{b+1}, \mathbf{Y}_{b+1}\right) \in A_{\epsilon}^{*(n)}\right\}
\end{aligned}
$$

Recalling that $\hat{u}_{1} \neq u_{1, b}$, we can bound

$$
\operatorname{Pr}\left\{E_{2}^{d} \mid\left(E_{1}^{d}\right)^{c}\right\} \leq \sum_{\hat{u}_{1} \in \mathcal{U}_{1}, \hat{u}_{1} \neq u_{1, b}} \operatorname{Pr}\left\{E_{2}^{d}\left(\hat{u}_{1}\right) \mid\left(E_{1}^{d}\right)^{c}\right\} .
$$

Using [36, Thm. 14.2.3], $\operatorname{Pr}\left\{E_{2}^{d}\left(\hat{u}_{1}\right) \mid\left(E_{1}^{d}\right)^{c}\right\}$ can be bounded by

$$
\begin{aligned}
\operatorname{Pr}\{ & \left.E_{2}^{d}\left(\hat{u}_{1}\right) \mid\left(E_{1}^{d}\right)^{c}\right\} \\
& \leq 2^{-n\left[I\left(V_{1}, X_{1}, X_{3} ; Y \mid S_{1}, S_{2}, T, V_{2}, X_{2}, W, Q\right)-\epsilon_{0}\right]} \\
& \stackrel{(a)}{=} 2^{-n\left[I\left(X_{1}, X_{3} ; Y \mid S_{1}, V_{2}, X_{2}, Q\right)-\epsilon_{0}\right]}
\end{aligned}
$$

where (a) follows from the Markov chains $V_{1}-\left(X_{1}, X_{2}, X_{3}\right.$, $\left.S_{1}, S_{2}, W, T, Q, V_{2}\right)-Y$ and $\left(S_{2}, T, W\right)-\left(X_{1}, X_{2}, X_{3}, S_{1}\right.$, $\left.V_{2}, X_{2}, Q\right)-Y$. Plugging (B.41) into B.40) we have

$$
\operatorname{Pr}\left\{E_{2}^{d} \mid\left(E_{1}^{d}\right)^{c}\right\} \leq 2^{n\left[R_{1}-\left(I\left(X_{1}, X_{3} ; Y \mid S_{1}, X_{2}, V_{2}, Q\right)-\epsilon_{0}\right)\right]},
$$

which can be bounded by $\epsilon$, for large enough $n$, as long as

$$
R_{1}<I\left(X_{1}, X_{3} ; Y \mid S_{1}, X_{2}, V_{2}, Q\right)-\epsilon_{0} .
$$

Following similar arguments as in B.39-B.43, we can also show that $\operatorname{Pr}\left\{E_{3}^{d} \mid\left(E_{1}^{d}\right)^{c}\right\}$ can be bounded by $\epsilon$, for large enough $n$, as long as

$$
R_{2}<I\left(X_{2}, X_{3} ; Y \mid S_{2}, X_{1}, V_{1}, Q\right)-\epsilon_{0},
$$

and $\operatorname{Pr}\left\{E_{4}^{d} \mid\left(E_{1}^{d}\right)^{c}\right\}$ can be bounded by $\epsilon$, for large enough $n$, as long as

$$
R_{1}+R_{2}<I\left(X_{1}, X_{2}, X_{3} ; Y \mid S_{1}, S_{2}, Q\right)-\epsilon_{0} .
$$

Hence, if conditions B.43 B.45 hold, for large enough $n, \bar{P}_{d, c h}^{(n)} \leq 5 \epsilon$.

Source decoder: From the SW theorem [12] it follows that, given correct decoding of $\left(u_{1, b}, u_{2, b}\right)$, the average probability of error in decoding $\left(\mathbf{s}_{1, b}, \mathbf{s}_{2, b}\right)$ at the destination can be made arbitrarily small for sufficiently large $n$, as long as

$$
\begin{aligned}
& H\left(S_{1} \mid S_{2}, W\right)+\epsilon_{0}<R_{1}, \\
& H\left(S_{2} \mid S_{1}, W\right)+\epsilon_{0}<R_{2}, \\
& H\left(S_{1}, S_{2} \mid W\right)+\epsilon_{0}<R_{1}+R_{2} .
\end{aligned}
$$

$$
\begin{aligned}
& \bar{P}_{d, c h}^{(n)} \triangleq \sum_{\left(\mathbf{s}_{1, b+1}, \mathbf{s}_{2}, b+1\right) \in \mathcal{S}_{1}^{n} \times \mathcal{S}_{2}^{n}} p\left(\mathbf{s}_{1, b+1}, \mathbf{s}_{2, b+1}\right) \sum_{\left(u_{1, b}, u_{2, b}\right) \in \mathcal{U}_{1} \times \mathcal{U}_{2}} p\left(u_{1, b}, u_{2, b}\right) \operatorname{Pr}\left\{E_{c h}^{d}\left(u_{1, b}, u_{2, b} ; \mathbf{s}_{1, b+1}, \mathbf{s}_{2, b+1}\right)\right\} \\
& \stackrel{(a)}{\leq} \epsilon+\quad \sum p\left(\mathbf{s}_{1, b+1}, \mathbf{s}_{2, b+1}, \mathbf{w}_{b+1}\right) \times \\
& \left(\mathbf{s}_{1, b+1}, \mathbf{s}_{2, b+1}, \mathbf{w}_{b+1}, \mathbf{t}_{b+1}\right) \in A_{\epsilon}^{*(n)}\left(S_{1}, S_{2}, W, T\right) \\
& \sum_{\left(u_{1, b}, u_{2, b}\right) \in \mathcal{U}_{1} \times \mathcal{U}_{2}} p\left(u_{1, b}, u_{2, b}\right) \operatorname{Pr}\left\{E_{c h}^{d}\left(u_{1, b}, u_{2, b} ; \mathbf{s}_{1, b+1}, \mathbf{s}_{2, b+1}\right) \mid \mathscr{D}_{b+1}\right\} .
\end{aligned}
$$


Combining conditions (B.43)-(B.45) with conditions $(B .46)$ yields the destination decoding constrains $30 \mathrm{e}-30 \mathrm{~g}$ in Thm. 7

\section{APPENDIX C \\ PROOF OF THEOREM 8}

Fix a distribution $p\left(s_{1}, s_{2}, w_{3}, w\right) p(q) p\left(x_{1} \mid s_{1}, q\right) p\left(x_{2} \mid s_{2}, q\right)$ $p\left(x_{3} \mid s_{1}, s_{2}, q\right) p\left(y_{3}, y \mid x_{1}, x_{2}, x_{3}\right)$.

\section{A. Codebook construction}

For $i=1,2$, assign every $\mathbf{s}_{i} \in \mathcal{S}_{i}^{n}$ to one of $2^{n R_{i}}$ bins independently according to a uniform distribution on $\mathcal{U}_{i} \triangleq$ $\left\{1,2, \ldots, 2^{n R_{i}}\right\}$. Denote this assignment by $f_{i}, i=1,2$.

For each $\mathbf{t} \in \mathcal{T}^{n}$ generate one $n$-length codeword $\mathbf{q}(\mathbf{t})$ by choosing the letters $q_{k}$ independently with distribution $p_{Q}\left(q_{k}\right)$, for $k=1,2, \ldots, n$. For each pair $\left(u_{i}, \mathbf{s}_{i}\right) \in \mathcal{U}_{i} \times$ $\mathcal{S}_{i}^{n}, i=1,2$, set $\mathbf{t}=h_{i}\left(\mathbf{s}_{i}\right)$, and generate one $n$-length codeword $\mathbf{x}_{i}\left(u_{i}, \mathbf{s}_{i}, \mathbf{q}(\mathbf{t})\right), \mathbf{s}_{i} \in \mathcal{S}_{i}^{n}, \mathbf{q} \in \mathcal{Q}^{n}$, by choosing the letters $x_{i, k}\left(u_{i}, \mathbf{s}_{i}, \mathbf{q}(\mathbf{t})\right)$ independently with distribution $p_{X_{i} \mid S_{i}, Q}\left(x_{i, k} \mid s_{i, k}, q_{k}(\mathbf{t})\right)$ for $k=1,2, \ldots, n$. Finally, generate one length- $n$ relay codeword $\mathbf{x}_{3}\left(\mathbf{s}_{1}, \mathbf{s}_{2}, \mathbf{q}(\mathbf{t})\right)$ for each pair $\left(\mathbf{s}_{1}, \mathbf{s}_{2}\right) \in \mathcal{S}_{1}^{n} \times \mathcal{S}_{2}^{n}$ by choosing $x_{3, k}\left(\mathbf{s}_{1}, \mathbf{s}_{2}, \mathbf{q}(\mathbf{t})\right)$ independently with distribution $p_{X_{3} \mid S_{1}, S_{2}, Q}\left(x_{3, k} \mid s_{1, k}, s_{2, k}, q_{k}(\mathbf{t})\right)$ for $k=1,2, \ldots, n$.

\section{B. Encoding}

Consider the sequences $s_{i, 1}^{B n} \in \mathcal{S}_{i}^{B n}, i=1,2, w_{3,1}^{B n} \in \mathcal{W}_{3}^{B n}$, and $w^{B n} \in \mathcal{W}^{B n}$, all of length $B n$. Partition each sequence into $B$ length- $n$ subsequences, $\mathbf{s}_{i, b}, i=1,2, \mathbf{w}_{3, b}$, and $\mathbf{w}_{b}, b=1,2, \ldots, B$. A total of $B n$ source samples are transmitted in $B+1$ blocks of $n$ channel symbols each. Let $\left(\mathbf{a}_{1}, \mathbf{a}_{2}\right) \in \mathcal{S}_{1}^{n} \times \mathcal{S}_{2}^{n}$ be two sequences generated i.i.d according to $p\left(\mathbf{a}_{1}, \mathbf{a}_{2}\right)=\prod_{k=1}^{n} p_{S_{1}, S_{2}}\left(a_{1, k}, a_{2, k}\right)$. These sequences are known to all nodes. At block 1 , source terminal $i, i=1,2$, observes $\mathbf{s}_{i, 1}$, finds its corresponding bin index $u_{i, 1}=f_{i}\left(\mathbf{s}_{i, 1}\right) \in$ $\mathcal{U}_{i}$, and transmits the channel codeword $\mathbf{x}_{i}\left(u_{i, 1}, \mathbf{a}_{i}, \mathbf{q}\left(h_{i}\left(\mathbf{a}_{i}\right)\right)\right)$. At block $b, b=2, \ldots, B$, source terminal $i, i=1,2$, transmits the channel codeword $\mathbf{x}_{i}\left(u_{i, b}, \mathbf{s}_{i, b-1}, \mathbf{q}\left(h_{i}\left(\mathbf{s}_{i, b-1}\right)\right)\right)$, where $u_{i, b}=f_{i}\left(\mathbf{s}_{i, b}\right) \in \mathcal{U}_{i}$. At block $B+1$, source terminal $i, i=1,2$, transmits $\mathbf{x}_{i}\left(1, \mathbf{s}_{i, B}, \mathbf{q}\left(h_{i}\left(\mathbf{s}_{i, B}\right)\right)\right)$.

Let $\mathbf{t}_{1}=h_{1}\left(\mathbf{a}_{1}\right)=h_{2}\left(\mathbf{a}_{2}\right)$. At block $b=1$, the relay transmits $\mathbf{x}_{3}\left(\mathbf{a}_{1}, \mathbf{a}_{2}, \mathbf{q}\left(\mathbf{t}_{1}\right)\right)$. Assume that at block $b, b=$ $2, \ldots, B, B+1$, the relay has estimates $\tilde{\mathbf{s}}_{i, b-1}$ of $\mathbf{s}_{i, b-1}, i=$ 1,2 , and let $\tilde{\mathbf{t}}_{b-1}=h_{1}\left(\tilde{\mathbf{s}}_{1, b-1}\right)=h_{2}\left(\tilde{\mathbf{s}}_{2, b-1}\right)$. The relay then transmits the channel codeword $\mathbf{x}_{3}\left(\tilde{\mathbf{s}}_{1, b-1}, \tilde{\mathbf{s}}_{2, b-1}, \mathbf{q}\left(\tilde{\mathbf{t}}_{b-1}\right)\right)$.

\section{Decoding}

The relay decodes the source sequences sequentially trying to reconstruct source block $\mathbf{s}_{i, b}, i=1,2$, at the end of channel block $b$ as follows: Let $\left(\tilde{\mathbf{s}}_{1, b-1}, \tilde{\mathbf{s}}_{2, b-1}\right)$ be the estimates of $\left(\mathbf{s}_{1, b-1}, \mathbf{s}_{2, b-1}\right)$ at the end of block $b-1$, and let $\tilde{\mathbf{t}}_{b-1} \triangleq$ $h_{1}\left(\tilde{\mathbf{s}}_{1, b-1}\right)=h_{2}\left(\tilde{\mathbf{s}}_{2, b-1}\right)$. The relay channel decoder at time $b$ decodes $\left(u_{1, b}, u_{2, b}\right)$, by looking for a unique pair $\left(\tilde{u}_{1}, \tilde{u}_{2}\right) \in$
$\mathcal{U}_{1} \times \mathcal{U}_{2}$ such that:

$$
\begin{aligned}
& \left(\tilde{\mathbf{s}}_{1, b-1}, \tilde{\mathbf{s}}_{2, b-1}, \tilde{\mathbf{t}}_{b-1}, \mathbf{q}\left(\tilde{\mathbf{t}}_{b-1}\right),\right. \\
& \quad \mathbf{x}_{1}\left(\tilde{u}_{1}, \tilde{\mathbf{s}}_{1, b-1}, \mathbf{q}\left(\tilde{\mathbf{t}}_{b-1}\right)\right), \mathbf{x}_{2}\left(\tilde{u}_{2}, \tilde{\mathbf{s}}_{2, b-1}, \mathbf{q}\left(\tilde{\mathbf{t}}_{b-1}\right)\right), \\
& \left.\quad \mathbf{x}_{3}\left(\tilde{\mathbf{s}}_{1, b-1}, \tilde{\mathbf{s}}_{2, b-1}, \mathbf{q}\left(\tilde{\mathbf{t}}_{b-1}\right)\right), \mathbf{w}_{3, b-1}, \mathbf{y}_{3, b}\right) \\
& \quad \in A_{\epsilon}^{*(n)}\left(S_{1}, S_{2}, T, Q, X_{1}, X_{2}, X_{3}, W_{3}, Y_{3}\right) .
\end{aligned}
$$

The decoded bin indices, denoted $\left(\tilde{u}_{1, b}, \tilde{u}_{2, b}\right)$, are then given to the relay source decoder, which estimates $\left(\mathbf{s}_{1, b}, \mathbf{s}_{2, b}\right)$ by looking for a unique pair of sequences $\left(\tilde{\mathbf{s}}_{1}, \tilde{\mathbf{s}}_{2}\right) \in$ $\mathcal{S}_{1}^{n} \times \mathcal{S}_{2}^{n}$ that satisfies $f_{1}\left(\tilde{\mathbf{s}}_{1}\right)=\tilde{u}_{1, b}, f_{2}\left(\tilde{\mathbf{s}}_{2}\right)=\tilde{u}_{2, b}$, and $\left(\tilde{\mathbf{s}}_{1}, \tilde{\mathbf{s}}_{2}, \mathbf{w}_{3, b}\right) \in A_{\epsilon}^{*(n)}\left(S_{1}, S_{2}, W_{3}\right)$. Denote the decoded sequences by $\left(\tilde{\mathbf{s}}_{1, b}, \tilde{\mathbf{s}}_{2, b}\right)$.

Decoding at the destination is done using backward decoding. The destination node waits until the end of channel block $B+1$. It first tries to decode $\mathbf{s}_{i, B}, i=1,2$, using the received signal at channel block $B+1$ and its side information $\mathbf{w}_{B}$. Going backwards from the last channel block to the first, at channel block $b$ we assume that the destination has estimates $\left(\hat{\mathbf{s}}_{1, b+1}, \hat{\mathbf{s}}_{2, b+1}\right)$ of $\left(\mathbf{s}_{1, b+1}, \mathbf{s}_{2, b+1}\right)$ and consider decoding of $\left(\mathbf{s}_{1, b}, \mathbf{s}_{2, b}\right)$. From $\left(\hat{\mathbf{s}}_{1, b+1}, \hat{\mathbf{s}}_{2, b+1}\right)$ the destination finds the corresponding $\left(\hat{u}_{1, b+1}, \hat{u}_{2, b+1}\right)$. Then, the destination decodes $\left(\mathbf{s}_{1, b}, \mathbf{s}_{2, b}\right)$ by looking for a unique pair $\left(\hat{\mathbf{s}}_{1}, \hat{\mathbf{s}}_{2}\right) \in \mathcal{S}_{1}^{n} \times \mathcal{S}_{2}^{n}$ such that:

$$
\begin{aligned}
& \left(\hat{\mathbf{s}}_{1}, \hat{\mathbf{s}}_{2}, \hat{\mathbf{t}}, \mathbf{q}(\hat{\mathbf{t}}), \mathbf{x}_{1}\left(\hat{u}_{1, b+1}, \hat{\mathbf{s}}_{1}, \mathbf{q}(\hat{\mathbf{t}})\right),\right. \\
& \left.\mathbf{x}_{2}\left(\hat{u}_{2, b+1}, \hat{\mathbf{s}}_{2}, \mathbf{q}(\hat{\mathbf{t}})\right), \mathbf{x}_{3}\left(\hat{\mathbf{s}}_{1}, \hat{\mathbf{s}}_{2}, \mathbf{q}(\hat{\mathbf{t}})\right), \mathbf{w}_{b}, \mathbf{y}_{b+1}\right) \\
& \quad \in A_{\epsilon}^{*(n)}\left(S_{1}, S_{2}, T, Q, X_{1}, X_{2}, X_{3}, W, Y\right) .
\end{aligned}
$$

where $\hat{\mathbf{t}}=h_{1}\left(\hat{\mathbf{s}}_{1}\right)=h_{2}\left(\hat{\mathbf{s}}_{2}\right)$. Denote the decoded sequences by $\hat{\mathbf{s}}_{1, b}$ and $\hat{\mathbf{s}}_{2, b}$.

\section{Error probability analysis}

Following arguments similar to those in Appendix B-D it can be shown that decoding the source sequences at the relay can be done reliably as long as (32a)- $-32 \mathrm{c}$ ) hold, and decoding the source sequences at the destination can be done reliably as long as $32 \mathrm{~d}-(32 \mathrm{~g})$ hold.

\section{REFERENCES}

[1] G. Kramer and A. J. Wijnngaarden, "On the white Gaussian multipleaccess relay channel". Proc. IEEE Int. Symp. Inform. Theory, Sorrento, Italy, Jun. 2000, p. 40.

[2] G. Kramer, M. Gastpar, and P. Gupta, "Cooperative strategies and capacity theorems for relay networks". IEEE Trans. Inform. Theory, vol. 51, no. 9, pp. 3037-3063, Sep. 2005.

[3] L. Sankaranarayanan, G. Kramer, and N. B. Mandayam, "Offset encoding for multiaccess relay channels". IEEE Trans. Inform. Theory, vol. 53, no. 10, pp. 3814-3821, Oct. 2007.

[4] R. Tandon and H. V. Poor, "On the capacity region of multiple-access relay channels". Proc. Conf. Inform. Sci. Syst., Baltimore, MD, Mar. 2011, pp. 1-5.

[5] C. E. Shannon, "A mathematical theory of communication". Bell Syst. Tech. J., vol. 27, pp. 379-423 and pp. 623-656, 1948.

[6] C. E. Shannon, "Two-way communication channels". Proc. 4th Berkeley Symp. Math. Statist. and Prob., vol. 1, pp. 611-644, 1961.

[7] T. M. Cover, A. El. Gamal, and M. Salehi, "Multiple access channels with arbitrarily correlated sources". IEEE Trans. Inform. Theory, vol. 26, no. 6, pp. 648-657, Nov. 1980.

[8] D. Gündüz, E. Erkip, A. Goldsmith, and H. V. Poor, "Source and channel coding for correlated sources over multiuser channels". IEEE Trans. Inform. Theory, vol. 55, no. 9, pp. 3927-3944, Sep. 2009. 
[9] T. M. Cover and A. A. El Gamal. "Capacity theorems for the relay channel". IEEE Trans. Inform. Theory, vol. 25, no. 5, pp. 572-584, Sep. 1979.

[10] E. Tuncel, "Slepian-Wolf coding over broadcast channels". IEEE Trans. Inform. Theory, vol. 52, no. 4, pp. 1469-1482, Apr. 2006.

[11] L. Sankar, N. B. Mandayam, and H. V. Poor, "On the sum-capacity of the degraded Gaussian multiaccess relay channel". IEEE Trans. Inform. Theory, vol. 55, no. 12, pp. 5394-5411, Dec. 2009.

[12] D. Slepian and J. K. Wolf, "Noiseless coding of correlated information sources". IEEE Trans. Inform. Theory, vol. 19, no. 4, pp. 471-480, Jul. 1973.

[13] L. Sankaranarayanan, G. Kramer, and N. B. Mandayam, "Capacity theorems for the multiple-access relay channel". Proc. 42nd Annu. Allerton Conf. Communications, Control, and Computing, Monticello, IL, Sep. 2004, pp. 1782-1791.

[14] S. Shamai (Shitz) and S. Verdú, "Capacity of channels with side information". Europ. Trans. Commun., vol. 6, no. 5, pp. 587-600, Sep. 1995.

[15] G. Dueck, "A note on the multiple access channel with correlated sources". IEEE Trans. Inform. Theory, vol. 27, no. 2, pp. 232-235, Mar. 1981.

[16] R. Ahlswede and T. S. Han, "On source coding with side information via a multiple access channel and related problems in multiuser information theory". IEEE Trans. Inform. Theory, vol. 29, no. 3, pp. 396-412, May 1983.

[17] T. S. Han and M. H. M. Costa, "Broadcast channels with arbitrarily correlated sources". IEEE Trans. Inform. Theory, vol. 33, no. 5, pp. 641-650, Sep. 1987.

[18] G. Kramer and C. Nair, "Comments on broadcast channels with arbitrarily correlated sources". Proc. IEEE Int. Symp. Inform. Theory, Seoul, Korea, Jul. 2009, pp. 2777-2779.

[19] M. Salehi and E. Kurtas, "Interference channels with correlated sources". Proc. IEEE Int. Symp. Inform. Theory, San Antonio, TX, Jan. 1993, p. 208.

[20] T. Han and K. Kobayashi, "A new achievable rate region for the interference channel". IEEE Trans. Inform. Theory, vol. 27, no. 1, pp. 49-60, Jan. 1981.

[21] W. Liu and B. Chen, "Interference channels with arbitrarily correlated sources". IEEE Trans. Inform. Theory, vol. 57, no. 12, pp. 8027-8037, Dec. 2011.

[22] N. Liu, D. Gündüz, A. Goldsmith and H. V. Poor, "Interference channels with correlated receiver side information". IEEE Trans. Inform. Theory, vol. 56, no. 12, pp. 5984-5998, Dec. 2010.

[23] D. Gündüz and E. Erkip, "Reliable cooperative source transmission with side information". Proc. IEEE Inform. Theory Workshop, Bergen, Norway, Jul. 2007, pp. 22-26.

[24] D. Gündüz, E. Erkip, A. Goldsmith and H. V. Poor, "Joint sourcechannel cooperative transmission over relay-broadcast networks". Accepted to the IEEE Trans. Inform. Theory, Oct. 2012.

[25] R. Kwak, W. Lee, A. El Gamal, and J. Cioffi, "Relay with side information". Proc. IEEE Int. Symp. Inform. Theory, Nice, France, Jun. 2007, pp. 606-610.

[26] M. Sefidgaran, B. Akhbari, Y. Mohsenzadeh and M. R. Aref, "Reliable source transmission over relay networks with side information". Proc. IEEE Int. Symp. Inform. Theory, Seoul, Korea, Jul. 2009, pp. 699-703.

[27] D. Gündüz and E. Erkip, "Joint source-channel codes for MIMO blockfading channels". IEEE Trans. Inform. Theory, vol. 54, no. 1, pp. 116134, Jan. 2008.

[28] S. Wu and Y. Bar-Ness. "OFDM systems in the presence of phase noise: consequences and solutions". IEEE Trans. Commun. vol. 52, no. 11, pp. 1988-1996 Nov. 2004.

[29] U. Erez, M. D. Trott, and G. W. Wornell, "Rateless coding and perfect rate-compatible codes for Gaussian channels". Proc. IEEE Int. Symp. Inform. Theory, Jul. 2006, Seattle, WA, pp. 528-532.

[30] B. Sklar, "Rayleigh fading channels in mobile digital communication systems part I: Characterization”. IEEE Commun. Mag., vol. 35, no. 7, pp. 90-100, Jul. 1997.

[31] R. Dabora. "The capacity region of the fading interference channel with a relay in the strong interference regime". IEEE Trans. Inform. Theory, vol. 58, no. 8, pp. 5172-5184, Aug. 2012.

[32] C. T. K. Ng, N. Jindal, A. J. Goldsmith, and U. Mitra, "Capacity gain from two-transmitter and two-receiver cooperation". IEEE Trans. Inform. Theory, vol. 53, no. 10, pp. 3822-3827, Oct. 2007.

[33] G. Farhadi and N. C. Beaulieu, "On the ergodic capacity of wireless relaying systems over Rayleigh fading channels". IEEE Trans. Wireless Commun., vol. 7, no. 11, pp. 4462-4467, Nov. 2008.
[34] G. Farhadi and N. C. Beaulieu, "On the ergodic capacity of multi-hop wireless relaying systems". IEEE Trans. Wireless Commun., vol. 8, no. 5, pp. 2286-2291, May 2009.

[35] I. Stanojev, O. Simeone, Y. Bar-Ness, and C. You, "Performance of multi-relay collaborative hybrid-ARQ protocols over fading channels". IEEE Commun. Lett., vol. 10, no. 7, pp. 522-524, Jul. 2006.

[36] T. M. Cover and J. Thomas, Elements of Information Theory. John Wiley and Sons Inc., 1991.

[37] R. W. Yeung, A First Course in Information Theory. Springer, 2002.

[38] F. D. Nesser and J. L. Massey, "Proper complex random processes with applications to information theory". IEEE Trans. Inform. Theory, vol 39. no. 4, pp. 1293-1302, Jul. 1993.

[39] A. El. Gamal and Y. H. Kim, "Network Information Theory". Cambridge University Press, 2012.

[40] J. L. Massey, "Causality, feedback and directed information". Proc. IEEE Int. Symp. Inform. Theory Appl., Nov. 1990, Waikiki, HI, pp. 303-305.

[41] Y. Oohama, "Gaussian multiterminal source coding". IEEE Trans. Inform. Theory, vol. 43, no. 6, pp. 1912-1923, Nov. 1997.

[42] M. Abramowitz and I. A. Stegun, Exponential Integral and Related Functions. Handbook of Mathematical Functions with Formulas, Graphs, and Mathematical Tables, 9th printing. New York: Dover, 1972.

[43] F. M. J.Willems. "Informationtheoretical Results for the Discrete Memoryless Multiple Access Channel". Doctor in de Wetenschappen Proefschrift dissertation, Katholieke Univ. Leuven, Leuven, Belgium, Oct. 1982.

[44] P. Gács and J. Körner, "Common information is much less than mutual information”. Probl. Contr. Inf. Theory, vol. 2, pp. 149-162, 1973.

[45] H. S. Witsenhausen, "On sequences of pairs of dependent random variables". SIAM J. Appl. Math., vol. 28, pp. 100-113, Jan. 2012. 